\title{
EFECTOS DE ACTIVIDADES HUMANAS EXTRACTIVAS Y SERVICIOS RECREATIVOS SOBRE LA CALIDAD DEL AGUA
}

Marco Antonio Aguirre

Doctorando en ciencias ambientales

Universidad del Valle Cali - Colômbia

marco.aguirre@correounivalle.edu.co https://orcid.org/0000-0002-5198-1748

\author{
Anderson Guillermo Rojas \\ Graduação en Geografia \\ Universidad del Valle Cali-Colômbia \\ andersongeografiauv@gmail.com; https://orcid.org/0000-0002-4636-7022
}

\section{Oscar Buitrago Bermúdez}

Doctor em Geografia

Universidad del Valle Cali-Colômbia

oscar.buitrago@correounivalle.edu.co; https://orcid.org/0000-0002-1726-9888

\author{
Francy Viviana Bolaños Trochez \\ Doctorando en ciencias ambientales \\ Universidad del Valle Cali - Colômbia \\ 1linfko2@gmail.com; https://orcid.org/0000-0001-8317-3553
}

\section{RESUMEN}

El artículo tiene como objetivo identificar algunos efectos de actividades humanas productivas de tipo extractivo y servicios recreativos — presentes en coberturas de la tierra - sobre la calidad del agua de la cuenca hidrográfica del río Dagua, localizada al suroccidente del departamento del Valle del Cauca, Colombia. Metodológicamente, el artículo integra información cuantitativa y cualitativa a través de seis fases: primero, definición de puntos de monitoreo de parámetros fisicoquímicos del agua y de aforo de caudales, así como áreas de trabajo para el cálculo de coberturas de la tierra; segundo, registro de parámetros fisicoquímicos del agua; tercero, construcción de índices síntesis de los parámetros fisicoquímicos; cuarto, identificación mediante trabajo en campo e información secundaria de actividades productivas de tipo extractivo y de servicios más representativas en la cuenca y, cálculo de porcentajes de coberturas de la tierra mediante información espacial a escala 1:100.000; quinto, evaluación de los efectos de las actividades productivas presentes en coberturas de la tierra sobre la calidad del agua mediante la técnica de panel de expertos; sexto, integración de los resultados de todas las fases. Entre los principales resultados de la investigación, se evidenció que la baja calidad ambiental del agua está asociada con actividades extractivas como la ganadería extensiva en ladera, la agricultura tecnificada, la minería extractiva (material de arrastre) sí mismo, que las actividades de servicios asociadas a la oferta de viviendas campestres para recreación y esparcimiento afectan la calidad y disponibilidad del agua en la cuenca.

Palabras clave: Actividades productivas. Calidad ambiental del agua. Cuenca hidrográfica río Dagua. Índice síntesis de calidad del agua. Percepción de la calidad del agua.

\section{EFFECTS OF HUMAN EXTRACTIVE ACTIVITIES AND RECREATIONAL SERVICES ON WATER QUALITY}

\section{ABSTRACT}

This paper aims to identify the effects of extractivist productive human activities and recreational services - developed in land covers - on the water quality of the Dagua river watershed, located in the southwestern area of the Valle del Cauca department (Colombia). Methodologically, the article integrates quantitative and qualitative data through six phases: First, defining monitoring points for physicochemical water parameters and flow gauging, as well as of work areas for calculating environmental quality by land cover type; second, carrying out a physicochemical water parameters registry; third, building synthesis indexes of physicochemical parametrers; fourth, identifying the most representative extractive productive activities type and services in the watershed (through fieldwork and secondary data), calculating land cover percentages through spatial information on a 1:100,000 scale; fifth, assessing the impacts of productive activities present in land cover on water quality through the technique of a panel of experts; sixth, integrating all the methodological phases. The research showed that the low environmental quality of the water is associated with extractive activities such as extensive livestock farming on hillsides, technified agriculture, extractive mining (dragging material) likewise, service activities associated to the supply of country house for recreation and leisure, affect the quality and availability of water in the watershed.

Keywords: Environmental quality of water. Perception of water quality. Productive activities. Synthesis index of water quality. Watershed Dagua's River.

Data da submissão: $30 / 04 / 2020$

Data de aceite: $22 / 06 / 2020$ 
Efectos de atividades humanas extractivas y servicios recreativos sobre la calidad del agua

\section{INTRODUCCIÓN}

Dentro del volumen total de agua presente en el planeta, el 97,5\% se encuentra en mares y océanos, $2 \%$ en casquetes polares y el $0,5 \%$ representa el agua subterránea, de lagos y ríos (Cirelli y Du Mortier, 2005). Por lo anterior, la disponibilidad, calidad y acceso al agua superficial o subterránea representan uno de los temas más importantes en la gestión del vital líquido, así como de las agendas gubernamentales en el mundo (Castro, 2007; Sutadian et al., 2016; Medeiros et al., 2017). En Colombia, pese a que alrededor del $78 \%$ de la población tiene acceso al agua potable, en algunas cuencas hidrográficas se evidencian problemas de salud ambiental relacionados con su calidad para consumo humano, debido a deficiencias de planificación y uso (Fao, 2018).

Las actividades humanas tienen una localización específica en la superficie terrestre. Para el caso particular de la cuenca hidrográfica, dicho anclaje se materializa a través de los usos y actividades productivas sobre diversas coberturas de la tierra, las cuales corresponden a aspectos morfológicos y tangibles sobre la superficie terrestre que resultan importantes para comprender y analizar cambios y patrones espaciales producidos natural y antrópicamente. Por lo tanto, una misma cobertura puede soportar diversos usos y actividades tales como ganadería, minería, silvicultura, enclaves industriales, sistemas de vivienda, entre otros, los cuales se espera afecten la calidad ambiental del agua.

Por esta razón, velar por la calidad ambiental del agua conlleva a las respectivas autoridades ambientales en Colombia a disponer instrumentos de planificación y gestión, a través de los cuales teóricamente se orienten las actividades humanas presentes en la cuenca hidrográfica que repercuten en la calidad (Houngbo, 2018; Naciones Unidas, 2010; Camdessus et al., 2006). Además, dichos instrumentos de carácter institucional deben garantizar la participación consultiva ${ }^{1}$ de las comunidades en la gestión del agua. Como consecuencia de dicha desarticulación se percibe un notorio proceso de afectación de las actividades humanas sobre importantes ecosistemas en las cuencas hidrográficas de Colombia (Andrade et al., 2018) y especialmente en la cuenca del río Dagua (Universidad del Valle, 2016a).

En ese contexto, el trabajo presenta un proceso metodológico a partir de métodos cualitativos y cuantitativos, como constituye la construcción de índices síntesis de calidad físicoquímica del agua y valoración perceptiva a través de panel de expertos desarrollados con agentes sociales clave. El trabajo contribuye con su aplicación a determinar la correspondencia perceptual entre el desarrollo de actividades productivas de tipo extractivo y de servicios presentes en coberturas de la tierra, con la baja calidad ambiental del agua, así como en la generación de información para la gestión y planificación ambiental emprendidos por comunidades locales e instituciones públicas en contextos como el de Colombia.

\section{ELEMENTOS TEÓRICOS}

En la cultura Occidental la espacialidad de las actividades humanas, asociada a su localización y emplazamiento en la superficie terrestre, se basa en la cosmovisión antropocéntrica, la cual al colocar en el centro de atención la vida humana desprecia otras formas de vida convirtiéndolas en recursos (agua, suelo, vegetación, minerales, entre otros). Bajo este principio utilitarista, el ser humano produce mecanismos de control y abuso con el fin de expandirse y ejercer dominio de toda la superficie terrestre (Leff, 2007; Collingwood, 2006; Lussault, 2015). De este modo, a lo largo de la historia los humanos han transformado su entorno en distintos niveles de intensidad, según el modo de producción capitalista y sus órdenes políticos, produciendo afectaciones irreversibles en ecosistemas, inclusive afectando al mismo ser humano.

\footnotetext{
${ }^{1}$ En Colombia de acuerdo con el Decreto 1640 de 2012, capítulo V, la participación planificación de cuencas hidrográficas, es consultiva y no deliberativa.
} 
Asimismo, en las últimas décadas se ha evidenciado cómo el desarrollo de las actividades humanas ha tenido implicaciones en el equilibrio ambiental del planeta, por ejemplo, en la alteración del ciclo natural del agua, entendido como el proceso de transformación y circulación del agua en la tierra (atmósfera, hidrósfera y litosfera), que involucra aspectos como la precipitación y evapotranspiración, además, representa el "intercambio de masa (agua) y energía que se esconde y libera a través de procesos de transición de fase del agua" (Ideam², 1998, p. 25); sin embargo, la expansión de las actividades humanas como la deforestación, intensificación de la agricultura, crecimiento de la población y la urbanización han alterado su regulación natural y directamente las propiedades organolépticas, físicas, químicas, radiactivas y microbiológicas del agua (Segura, 2007; Mc Junkin, 1988; Carstens \& Amer, 2018; Pérez, Nardini y Galindo, 2018; Rojas y Campo, 2018).

En ese sentido, los antecedentes acerca de la relación entre coberturas de la tierra y calidad del agua en ríos permiten orientar metodológicamente el trabajo a partir de dos perspectivas: de tipo cuantitativo y cualitativo. Las primeras, buscan establecer grados de correspondencia entre calidad del agua con usos y coberturas, mediante técnicas estadísticas y monitoreo de parámetros físicoquímicos y biológicos, teniendo presente las precipitaciones anuales $\mathrm{y}$ mensuales. Metodológicamente se resalta, primero, la definición de áreas de trabajo o micro-cuencas, en las cuales se localizan puntos de monitoreo de calidad del agua. No obstante, hace falta precisar los criterios para la selección de dichas áreas, ya que buena parte de los estudios consultados se basan en la definición espacial arbitraria sin tener presente, al momento de su selección, criterios geográficos.

Segundo, en todos los casos indagados, se calcula el porcentaje de coberturas a través de geoprocesamiento de imágenes áreas y satelitales con software especializados (Zhao, et al., 2015; Robledo, et al., 2014; Guerreo, 2011; Li et al., 2008; Auquilla, 2005; Ahearn, et al., 2005). Tercero, se emplean índices de calidad del agua estandarizados a nivel mundial (ICAs) y, finalmente, mediante métodos estadísticos como el de correlación de Pearson, siendo este uno de los más empleados, se estima la relación entre coberturas de la tierra y calidad ambiental del agua (Zhou, et al., 2016; Acosta, 2015; Bu, et al., 2014; Gyawali et al., 2013).

Por otro lado, los estudios de carácter cualitativo buscan determinar desde la percepción y experiencia de los agentes sociales, las actividades humanas que afectan la calidad ambiental del agua (Benez., 2009; Tomaselli, 2013; Billen, et al., 2011; Samboni, et al., 2007; Fernandez y Solano, 2009; Londoño, 2001). A nivel metodológico, primero se identifican las actividades productivas y usos del suelo más importantes en una cuenca hidrográfica relacionadas con el agua (de tipo agropecuario, industrial, doméstico, de generación de energía, minería, producción de alimentos, usos recreativos, entre otros); y segundo, se diseñan y ejecutan entrevistas semiestructuradas para indagar sobre el grado de afectación de dichas actividades productivas en el agua (Loaiza y Osorio, 2009; Senzanje, et al., 2008; Boelee \& Laamrani, 2004). Adicionalmente, se destaca que una forma de reforzar este tipo de estudios es mediante la realización de talleres de cartografía social para evidenciar espacial y temporalmente cambios en la calidad ambiental del agua y el paisaje en cuencas hidrográficas (Auquilla, 2005; Aguirre et al., 2017; Universidad del Valle, 2016b).

A pesar de existir gran diversidad de trabajos cuantitativos y cualitativos para conocer los efectos de las actividades humanas sobre la calidad del agua, hace falta profundizar en métodos mixtos que permitan comprender, desde campos diversos, la complejidad que existe en una cuenca hidrográfica como unidad espacial donde se localizan y desarrollan diversas prácticas humanas. La integración de estas técnicas facilita la producción de información clave a los tomadores de decisiones ambientales y las comunidades locales para hacer frente a la gestión del agua (Tyagi et al., 2013; Sutadian et al., 2016; Pérez, Nardini y Galindo, 2018).

\footnotetext{
${ }^{2}$ Instituto de Hidrología, Meteorología y Estudios Ambientales (Ideam) de Colombia.
} 


\section{3 ÁREA DE ESTUDIO}

La cuenca del río Dagua hace parte de la porción central del denominado Andén Pacífico colombiano, el cual comprende fisiográficamente el sector occidental de Colombia, Ecuador y Panamá y desde el borde costero hasta la divisoria de aguas de la cordillera Occidental; área que al ser afectada por la Zona de Convergencia Intertropical (ZCI) presenta alta pluviosidad, lo cual sumado a su baja altitud y a su localización sobre la zona de subducción de la placa de Nazca, por debajo de la de Suramérica, la configuran como de enorme complejidad biogeográfica.

Debido a su compleja geodinámica que da origen al Andén Pacífico, las condiciones geomorfológicas evidencian abruptos cambios de topografía que se expresan en dos partes: alta montañosa, con procesos naturales de erosión del suelo, donde actualmente, se desarrollan actividades extractivas como agricultura y ganadería en ladera, cultivos forestales de pino y eucalipto, y parcelación acelerada del suelo para construcción de viviendas de recreo (Universidad del Valle, 2016a, 2016b); sus precipitaciones oscilan entre 900 y $1.600 \mathrm{~mm} / \mathrm{año}$; y parte baja, de relieve colinado y plano, donde predomina el bosque denso de tierra firme tropical, el cual evidencia procesos extractivos legales e ilegales de materiales de arrastre del cauce principal del río Dagua y minería aurífera artesanal; su precipitación oscila entre 6.000 y $8.000 \mathrm{~mm} /$ año (CVC, 2011.) (Ver Figura 1).

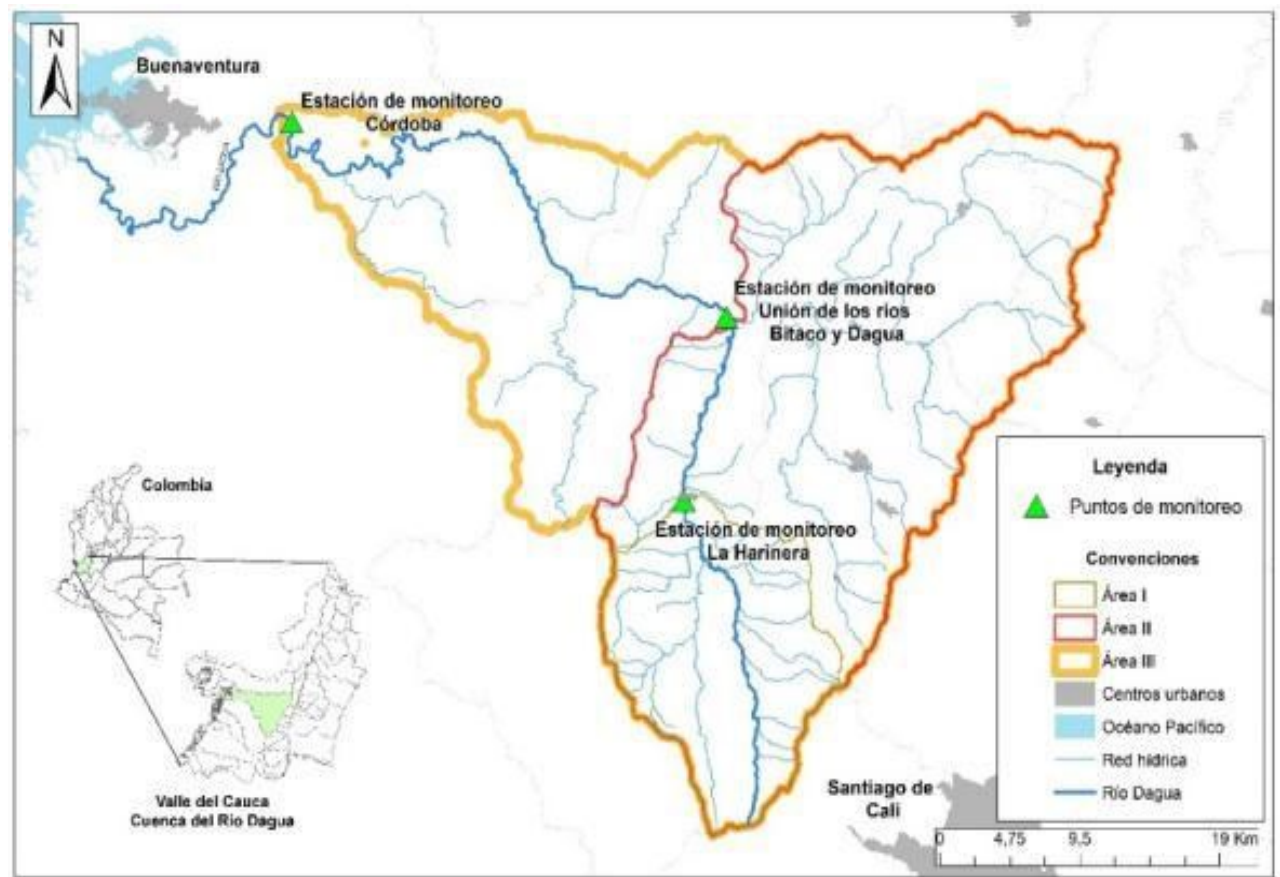

Figura 1. Localización de la cuenca hidrográfica del río Dagua y estaciones de monitoreo Datos tomados de la Universidad del Valle

Fuente: Elaborado por los autores

En la cuenca tienen anclaje espacial diversos agentes sociales, entre los cuales se destacan: comunidades afrodescendientes (consejos comunitarios); grupos indígenas (a través de resguardos); y campesinos, subdivididos entre pequeños productores (con prácticas agrícolas de subsistencia o pancoger) y, medianos productores (con mayores grados de tecnificación agropecuaria). Sus prácticas culturales y epistémicas inciden en las actividades económicas productivas que se desarrollan en las distintas coberturas de la tierra sobre la cuenca.

La cuenca hidrográfica es de interés nacional y regional por dos razones: primero, su ubicación geoestratégica con respecto al principal puerto marítimo de Colombia localizado en la bahía de Buenaventura sobre el océano Pacífico. Este elemento espacial convierte a la cuenca hidrográfica en un importante canal de intercambio de bienes, productos y servicios de importación 
y exportación que dinamizan la economía nacional (Buitrago, Bolaños \& Aguirre, 2017). En ella tienen injerencia espacial y territorial cinco municipios (Dagua, Restrepo, La Cumbre, Vijes y Buenaventura) los cuales, a la fecha, no han logrado acuerdos supramunicipales para la gestión coordinada del agua y de su calidad ambiental (Aguirre et al, 2017; Rojas y Campo, 2018).

\section{METODOLOGÍA}

La metodología integra seis fases (ver Figura 2).

Fase I: Tuvo como objetivo definir puntos de monitoreo para el registro de parámetros fisicoquímicos del agua, medición de caudales y selección de áreas aguas arriba de cada punto de monitoreo para estimar porcentajes de coberturas de la tierra.

Para definir los puntos de monitoreo, primero, se estimó, a partir de un modelo de elevación digital del terreno (DEM a 30 metros de resolución espacial), la red hídrica principal de la cuenca con sus puntos de aforo mediante el módulo de Arc GIS Arc Hydro Tools. La herramienta destaca elementos en la red hídrica asociados a puntos de intersección de afluentes de primer y segundo orden, a ellos les denomina puntos de aforo. Con los puntos de aforo y a través de criterios geográficos como el relieve y acceso a vías principales, se seleccionaron tres puntos de monitoreo sobre el cauce principal del río Dagua correspondientes a La Harinera, Unión de los ríos Dagua y Bitaco, localizados en la cuenca alta, y Córdoba, localizado en la cuenca baja (ver Figura 1).

Fase II. Para la toma de parámetros fisicoquímicos del agua se empleó una sonda multiparamétrica HANNA HI 9828, la cual registró los siguientes parámetros: Potencial OxidoReducción (\%ORP); $\mathrm{pH} ; \mathrm{pHmV}$; conductividad $\mu \mathrm{S} / \mathrm{cm}$; conductividad $\mu \mathrm{S} / \mathrm{cm}^{\mathrm{A}}$; salinidad; temperatura; presión atmosférica; Sólidos Totales Disueltos (TDS); \% de Saturación de Oxígeno (\%OD); Oxígeno Disuelto (OD ppm); y, Resistividad ${ }^{3}$. El registro se realizó durante cinco meses, de octubre 2016 a febrero 2017, los días jueves, viernes y sábado, en tres momentos del día, 7:00 am, 1:00 pm y 6:00 pm. Adicionalmente, se registró el caudal del río en cada punto de monitoreo con el fin de contrastar los índices síntesis fisicoquímicos con los flujos de agua.

Fase III: Los índices síntesis fisicoquímicos del agua se obtuvieron mediante Análisis Factorial Múltiple -AFM- (Escofier y Pagés, 1992), el cual permite el análisis simultáneo de varios grupos de variables medidas sobre el mismo conjunto de individuos equilibrando la influencia de cada grupo a una misma naturaleza (datos cualitativos o cuantitativos). A su vez, pondera cada grupo de variables para que tengan la misma importancia en la construcción del primer factor, el cual será el índice buscado por estación de monitoreo. Cabe mencionar que para asegurar la confiabilidad del AFM se determinó el factor tamaño y se observó la variabilidad del total de los datos. Los cálculos se realizaron con el software R, haciendo uso del paquete FactorMineR del cual se obtuvo el círculo de correlaciones, el factor tamaño y el componente principal por estación. A partir del primer componente principal se construyeron los índices síntesis fisicoquímicos para los tres puntos de monitoreo; es necesario aclarar que los índices obtenidos a partir del protocolo y la metodología expuesta, se aproximan más a los requerimientos para la conservación de la vida acuática, además de ser aplicables únicamente al río Dagua. Finalmente, la escala de medición va de 0 a 1 , donde cero corresponde a buen estado ambiental del agua.

Fase IV: Para identificar las actividades productivas más representativas en la cuenca, se partió del mapa de coberturas de la tierra suministrado por el -Ideam- a escala 1:100.000 correspondiente al año 2013, el cual usó la clasificación de Corine Land Cover ajustada para Colombia. A través de observaciones en campo, con el mapa de coberturas y mediante información secundaria producida por instituciones oficiales, se identificaron las actividades productivas más representativas por coberturas de la tierra. Posteriormente, con las actividades identificadas en cada cobertura se observaron patrones de regularidad espacial que condujeron al reagrupamiento de las

\footnotetext{
${ }^{3}$ No se incluyen parámetros biológicos debido a limitaciones de presupuesto.
} 
mismas en 5 conjuntos (Ver Figura 2). Con ello, se procedió al cálculo de porcentajes de coberturas de la tierra para cada una de las áreas de trabajo definidas en la Fase I.

\begin{tabular}{|c|c|}
\hline & Coberturas de la tierra* \\
\hline & Área total de la cuenca \\
\hline \multirow{5}{*}{ C1** } & Pastos limpios (ganadería en ladera) \\
\hline & Pastos enmalezados \\
\hline & Herbazal (herbazal denso tierra firme) \\
\hline & Arbustales \\
\hline & Mosaico de cultivos y espacios naturales \\
\hline $\mathrm{C} 2 * *$ & Área construida \\
\hline \multirow{5}{*}{$\mathrm{C}^{* * *}$} & Bosque denso \\
\hline & Bosque fragmentado (con pastos) \\
\hline & Bosque ripario \\
\hline & Vegetación secundaria en transición \\
\hline & Plantación forestal (pino y eucalipto) \\
\hline $\mathrm{C} 4 * *$ & Cultivos permanentes y semipermanentes (frutales cítricos, café, piña y sábila) \\
\hline C5** & $\begin{array}{l}\text { Mosaico de cultivos, cultivos arbustivos y transitorios y pan coger (Té, caña panelera, } \\
\text { frutales, maíz y hortalizas) }\end{array}$ \\
\hline No dato & Nubes y cuerpos de agua (ríos) \\
\hline
\end{tabular}

Figura 2. Coberturas de la tierra cuenca hidrográfica río Dagua.

Fuente: Elaborado por los autores

Fase V: Con el objetivo validar y complementar los resultados de la fase III, se indagó desde el saber consuetudinario por la percepción de los agentes sociales acerca de la afectación de las actividades productivas presentes en las coberturas de la tierra sobre la calidad del agua, para lo cual se aplicó la técnica de panel de expertos en la cuenca alta y baja del río Dagua. Los participantes fueron seleccionados mediante muestreo teórico o intencional (Martínez, 2012) teniendo como criterios: 1) la experiencia en procesos de gestión ambiental en la cuenca, 2) edades superiores a los 30 años y 3) personas (hombres y mujeres) dedicadas a actividades de tipo extractivo y de servicios recreativos. El panel de expertos integró las siguientes categorías: elementos biológicos, hidromorfológicos, físicos y organolépticos del agua (Figura 3). Para lo anterior, fue necesario la siguiente tipología: agentes institucionales, sociedad civil y usuarios del agua, (Lobato, 2014). En total, se contó con 40 participantes en el panel de expertos entre cuenca alta y baja.

\begin{tabular}{|c|c|c|c|}
\hline \multicolumn{2}{|c|}{ Categorías } & \multicolumn{2}{|l|}{ Aspectos } \\
\hline \multirow{3}{*}{$\begin{array}{c}\text { Factores } \\
\text { ambientales }\end{array}$} & \multirow{2}{*}{$\begin{array}{l}\text { Relativos a la } \\
\text { calidad del } \\
\text { agua }\end{array}$} & $\begin{array}{l}\text { Biológicos: organismos existentes en el agua, } \\
\text { importantes en la percepción de la calidad del } \\
\text { líquido. (Arruebo, T, et al. } 2009 \text { ) }\end{array}$ & $\begin{array}{l}\text {-Fauna } \\
\text {-Flora } \\
\text {-Vegetación de ribera }\end{array}$ \\
\hline & & $\begin{array}{l}\text { Hidromorfológicos: elementos físicos de las } \\
\text { riberas del río (Ferrer \& Muñoz, 2013) }\end{array}$ & $\begin{array}{l}\text {-Variación de la profundidad } \\
\text {-Cauce y caudal }\end{array}$ \\
\hline & $\begin{array}{l}\text { Factores } \\
\text { relativos a la } \\
\text { aceptabilidad } \\
\text { del agua }\end{array}$ & $\begin{array}{l}\text { Físicos y organolépticos: factores físicos que } \\
\text { inciden en aceptabilidad del agua que puede } \\
\text { ser subjetiva y afectada por factores que } \\
\text { resultan desagradables visualmente (OMS, } \\
2006 \text { ) }\end{array}$ & $\begin{array}{l}\text {-Olor } \\
\text {-Temperatura } \\
\text {-Transparencia }\end{array}$ \\
\hline \multicolumn{2}{|c|}{ Uso del agua } & $\begin{array}{l}\text { Se refiere al fin que se le está dando al agua } \\
\text { en cualquier actividad, desde su } \\
\text { disponibilidad hasta la salud de las personas } \\
\text { en el consumo }\end{array}$ & $\begin{array}{l}\text {-Disponibilidad } \\
\text {-Salud de las personas }\end{array}$ \\
\hline
\end{tabular}

Figura 3. Categorías de análisis utilizadas en el panel de expertos.

Fuente: Elaborado por los autores

Revista de Gestão Social e Ambiental - RGSA, São Paulo, v. 14, n. 2, p. 82-104, maio/ago. 2020. 
Para orientar la valoración del panel, se diseñó una escala numérica (Ver figura 4) que representa lo percibido en cuanto al grado de afectación de la relación existente, sea este positivo o negativo, entre las actividades productivas y la calidad del agua.

\begin{tabular}{|c|c|c|c|c|}
\hline No afecta (0) & Muy negativa (-2) & Negativa (-1) & Positiva (1) & Muy positiva (2) \\
\hline $\begin{array}{l}\text { No implica un uso } \\
\text { estricto del agua, } \\
\text { dicha actividad no } \\
\text { es representativa en } \\
\text { términos de } \\
\text { afectación. }\end{array}$ & $\begin{array}{l}\text { Aporta en alto grado } \\
\text { elementos que afectan } \\
\text { visiblemente la calidad } \\
\text { del agua, y su desarrollo } \\
\text { comprometer la } \\
\text { disponibilidad del } \\
\text { recurso. }\end{array}$ & $\begin{array}{l}\text { Afecta } \\
\text { moderadamente la } \\
\text { disponibilidad en las } \\
\text { fuentes de captación } \\
\text { y aporta algunos } \\
\text { elementos } \\
\text { perjudiciales. }\end{array}$ & $\begin{array}{l}\text { No aporta } \\
\text { elementos } \\
\text { que afecten el } \\
\text { agua durante } \\
\text { su uso. }\end{array}$ & $\begin{array}{l}\text { El uso del agua en tal } \\
\text { acción afecta } \\
\text { positivamente } \\
\text { calidad del agua. }\end{array}$ \\
\hline
\end{tabular}

Figura 4. Valoración numérica del grado de afectación del agua.

Fuente: Elaborado por los autores

Posteriormente, con el fin de sistematizar la información obtenida en los paneles, se elaboró una matriz de resultados a partir de un promedio final de las valoraciones numéricas correspondiente al grado de afectación, determinando los rangos que se pueden apreciar en el Figura 5.

\begin{tabular}{|c|c|c|c|c|c|}
\hline \multirow{4}{*}{ Acción } & \multicolumn{3}{|c|}{ Afectación } \\
\cline { 2 - 4 } & \multicolumn{2}{|c|}{ Positiva } & Nulo afecta & \multicolumn{2}{c|}{ Negativa } \\
\cline { 2 - 5 } & Bajo & Alto & \multirow{2}{*}{0} & Bajo & Alto \\
\cline { 2 - 5 } & 1 a 5 & Mayor a 5 & & -1 a -5 & Mayor a -5 \\
\hline
\end{tabular}

Figura 5. Rangos propuestos para el análisis paneles de expertos.

Fuente: Elaborado por los autores

Fase VI: Finalmente, se integró los resultados obtenidos del proceso metodológico para comprender la correspondencia perceptual entre índices síntesis de calidad fisicoquímicos del agua con actividades antrópicas de tipo extractivo y de servicios mediante porcentajes de coberturas de la tierra. Para ello, se elaboraron cinco gráficos con doble ordenada (eje y) por conjunto de coberturas; en la ordenada izquierda están los índices fisicoquímicos del agua de cada mes y, en la ordenada derecha, los porcentajes de coberturas aguas arriba de cada punto de monitoreo; en tanto que, en la abscisa (eje $\mathrm{x}$ ), se consideraron los tres puntos de monitoreo. 
Efectos de atividades humanas extractivas y servicios recreativos sobre la calidad del agua

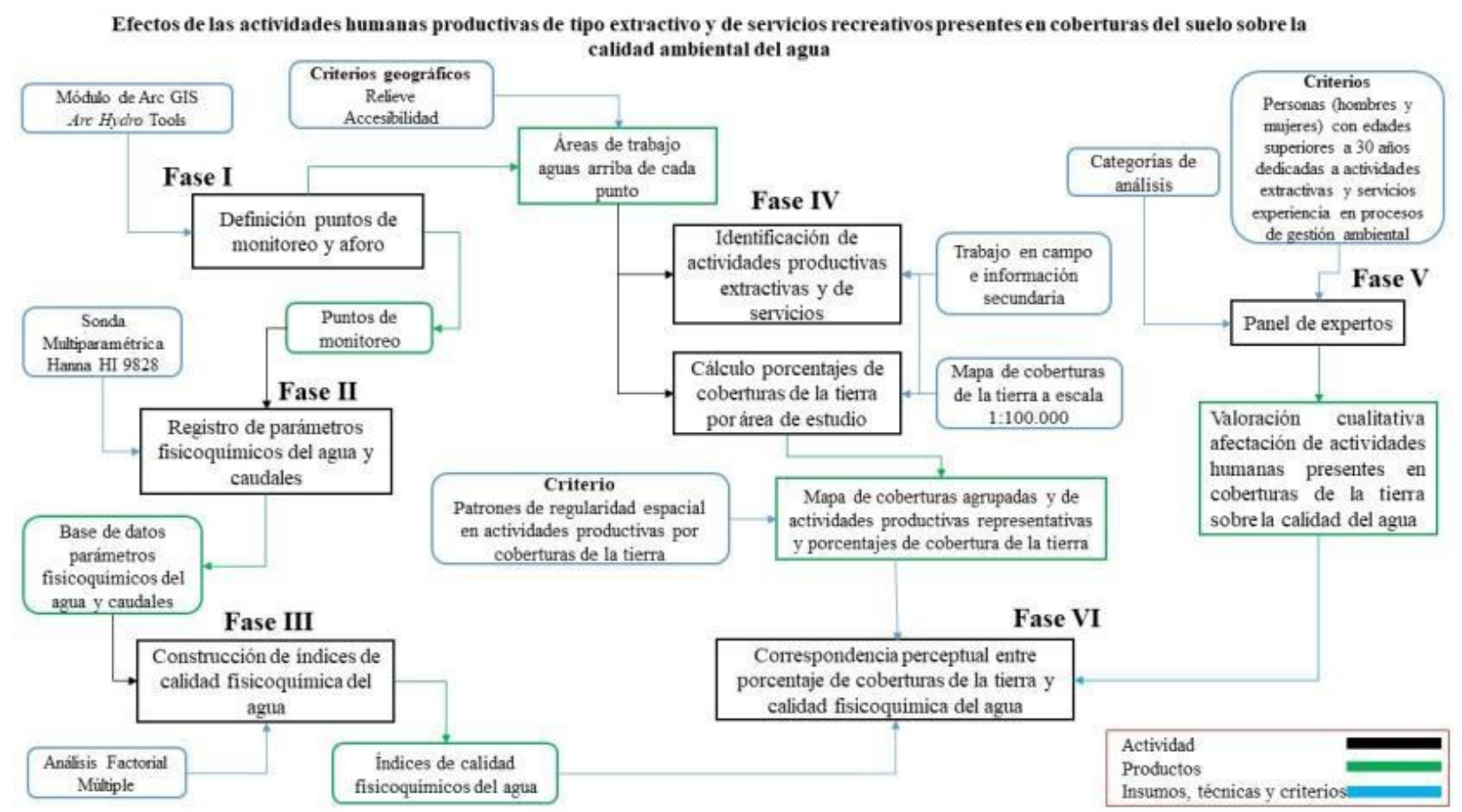

Figura 6. Metodología general.

Fuente: Elaborado por los autores

\section{DISCUSIÓN DE RESULTADOS}

\section{Definición de áreas de trabajo y puntos de monitoreo}

En el Figura 1 se observan los puntos de monitoreo definidos para llevar a cabo la toma de parámetros fisicoquímicos y caudales del agua. Así mismo, se presentan las áreas para el cálculo de coberturas de la tierra aguas arriba de cada punto.

\section{Índice síntesis del estado fisicoquímico del agua para el río Dagua}

En la Figura 7 se observa un diagrama de cajas con los parámetros fisicoquímicos estandarizados y sin estandarizar previo a la elaboración de la matriz de correlación. Seguidamente, la Figura 5 ilustra la matriz de correlación de los parámetros físico-químicos registrados sobre el cauce principal del río Dagua durante los cinco meses de muestreo.

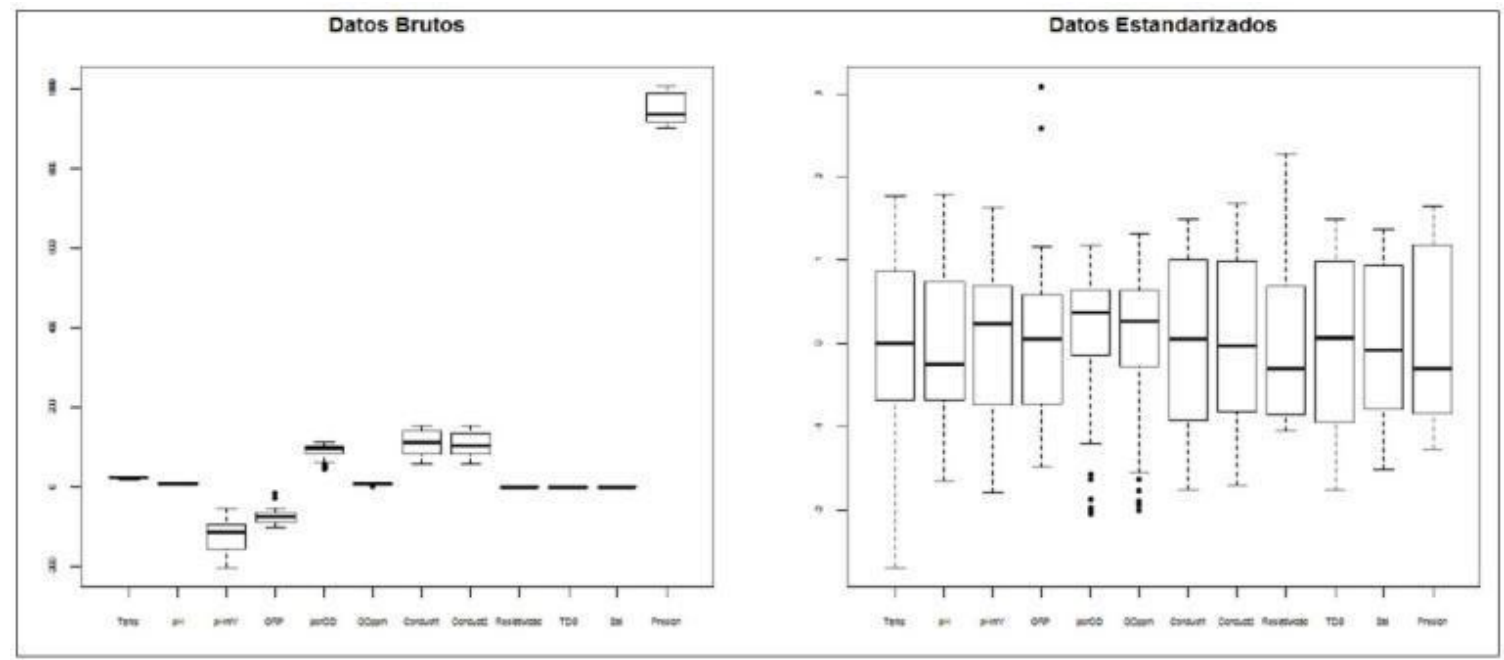

Figura 7. Estandarización de parámetros fisicoquímicos del agua.

Fuente: Elaborado por los autores 
Con los resultados de la matriz de correlación (ver Figura 8) se determinó los parámetros respectivos para el cálculo del índice: Temperatura $\left({ }^{\circ} \mathrm{C}\right), \mathrm{pH}$, porOD (\% oxígeno disuelto), TDS (solidos totales disueltos) y Conductividad $1(\mu \mathrm{S} / \mathrm{cm})$; y a su vez, se definió el factor tamaño previo a la construcción de índices como afirma Escobar (2008) (ver Figura 9).

\begin{tabular}{|c|c|c|c|c|c|c|c|c|c|c|c|c|}
\hline Parámetros & Temp & $\mathrm{pH}$ & $\mathrm{pHmV}$ & ORP & porOD & ODppm & Cond. 1 & Cond.2 & Resistivi & TDS & Sal & Presión \\
\hline Temp & 1 & & & & & & & & & & & \\
\hline $\mathrm{pH}$ & $-0,0457$ & 1 & & & & & & & & & & \\
\hline $\mathrm{pHmV}$ & 0,0290 & $-0,9998$ & 1 & & & & & & & & & \\
\hline ORP & $-0,3311$ & 0,4547 & $-0,4502$ & 1 & & & & & & & & \\
\hline porOD & $-0,0106$ & $-0,8436$ & 0.8447 & $-0,3759$ & 1 & & & & & & & \\
\hline ODppm & $-0,0320$ & -0.8560 & 0,8575 & -0.3216 & 0,9904 & 1 & & & & & & \\
\hline Conduct1 & $-0,3043$ & 0,4570 & $-0,4537$ & 0,0216 & $-0,3449$ & $-0,4134$ & 1 & & & & & \\
\hline Conduct2 & $-0,1958$ & 0.4650 & $-0,4631$ & -0.0182 & -0.3567 & $-0,4303$ & 0.9935 & 1 & & & & \\
\hline Resistividad & 0,3434 & $-0,5327$ & 0,5286 & $-0,0778$ & 0,3920 & 0,4664 & $-0,9637$ & $-0,9523$ & 1 & & & \\
\hline TDS & $-0,3043$ & 0,4581 & $-0,4544$ & 0,0228 & $-0,3469$ & $-0,4152$ & 0,9999 & 0,9953 & $-0,9632$ & 1 & & \\
\hline Sal & $-0,2942$ & 0,3624 & $-0,3486$ & $-0,0325$ & $-0,2571$ & $-0,3247$ & 0,9801 & 0,9744 & $-0,9231$ & 0,9805 & 1 & \\
\hline Presión & 0,5869 & $-0,3024$ & 0,2928 & $-0,0130$ & 0.1724 & 0,2642 & $-0,7032$ & $-0,6601$ & 0,7632 & $-0,7028$ & $-0,6705$ & 1 \\
\hline
\end{tabular}

Figura 8. Matriz de correlación

Fuente: Elaborado por los autores

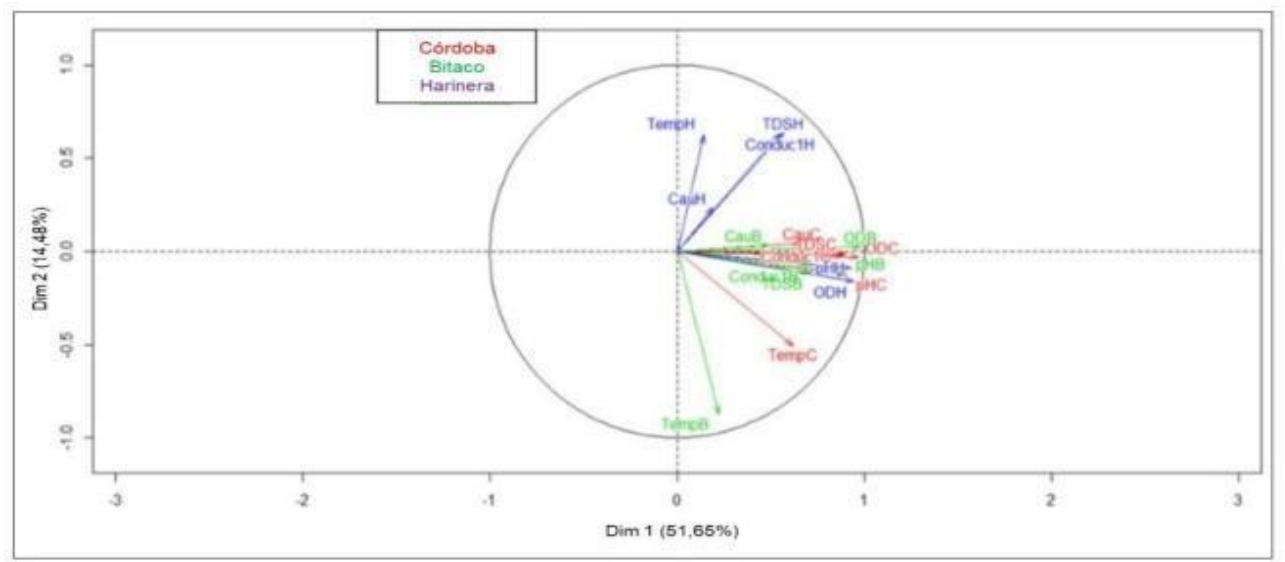

Figura 9. Circulo de Correlaciones para determinar factor tamaño de los índices.

Fuente: Elaborado por los autores

En la Figura 10 se observan los componentes principales obtenidos mediante la función del coseno cuadrado para las tres estaciones (Bitaco, Córdoba y La Harinera); es evidente en la suma total de los componentes que las variables con mayor representación corresponden a OD (Oxígeno Disuelto) y pH (Indica la acidez o alcalinidad). Así mismo, se muestra que la variable con menor representación es el Caudal. De esta manera, se obtuvieron los índices de calidad fisicoquímica del agua para las 3 estaciones. 


\begin{tabular}{|lccc|}
\hline & Comp 1 & Comp 2 & Suma \\
\hline TempC & 0.38 & 0.26 & 0.64 \\
pHC & 0.82 & 0.02 & $\mathbf{0 . 8 3}$ \\
ODC & 0.94 & 0.00 & $\mathbf{0 . 9 4}$ \\
Conduc1C & 0.79 & 0.00 & 0.79 \\
TDSC & 0.81 & 0.00 & 0.81 \\
CauC & 0.44 & 0.00 & 0.45 \\
TempB & 0.05 & 0.76 & 0.81 \\
pHB & 0.81 & 0.01 & $\mathbf{0 . 8 3}$ \\
ODB & 0.96 & 0.00 & $\mathbf{0 . 9 6}$ \\
Conduc1B & 0.51 & 0.01 & 0.51 \\
TDSB & 0.51 & 0.01 & 0.52 \\
CauB & 0.24 & 0.00 & 0.24 \\
TempH & 0.02 & 0.39 & 0.41 \\
pHH & 0.87 & 0.01 & $\mathbf{0 . 8 8}$ \\
ODH & 0.89 & 0.03 & $\mathbf{0 . 9 2}$ \\
Conduc1H & 0.30 & 0.40 & 0.70 \\
TDSH & 0.32 & 0.40 & 0.73 \\
CauH & 0.01 & 0.04 & 0.05 \\
\hline
\end{tabular}

Figura 10. Coseno cuadrado de las variables. $\mathrm{C}=$ Córdoba; $\mathrm{B}=$ Bitaco; $\mathrm{H}=$ Harinera. Fuente: Elaborado por los autores

Bajo el criterio de personal experto en calidad del agua y teniendo en cuenta literatura sobre niveles óptimos de los parámetros físicoquímicos medidos para el uso de conservación de vida acuática, se establecieron rangos para calificar la calidad del agua según los parámetros registrados. Como resultado se presenta el figura 11 cuyos valores de calificación van de 0 a 1 .

\begin{tabular}{|c|c|c|}
\hline \multicolumn{2}{|c|}{ Rangos propuestos para la interpretación del estado fisicoquímico del agua } \\
\hline Bueno & Medio & Malo \\
\hline $0-0.30$ & $0.31-0.46$ & $0.47-1$ \\
\hline
\end{tabular}

Figura 11: Rangos propuestos para la interpretación del estado fisicoquímico del agua.

Fuente: Elaborado por los autores

Los rangos establecidos permitieron el análisis de los índices síntesis fisicoquímicos del agua durante cinco meses para dos periodos distintos. El primero, caracterizado por altas precipitaciones entre los meses de octubre a diciembre del año 2016; el segundo, por bajas precipitaciones entre los meses de enero a marzo de 2017. De este modo, en la Figura 8 se observa que la calidad ambiental del agua, para el caso de las estaciones de monitoreo ubicadas en la cuenca alta, fue baja en la medida que disminuyeron las precipitaciones.

En el punto La Harinera, durante los meses de octubre y noviembre (color azul), el agua presentó un buen estado fisicoquímico, que para diciembre y enero disminuyó a un rango medio (color amarillo). Por su parte, el mes de febrero asociado a precipitaciones bajas, reflejó un mal estado de su calidad (color rojo). En el punto de monitoreo denominado Bitaco, durante los meses de octubre, diciembre y enero los índices de calidad ambiental se mantuvieron en un rango medio (color amarillo), siendo de baja calidad en febrero (color rojo) con excepción del mes de noviembre, que evidenció buena calidad ambiental del agua (color azul). Por otro lado, en la estación de Córdoba, ubicada en la cuenca baja, los índices de octubre, noviembre y diciembre presentaron buena y media calidad del agua (color azul y amarillo) distinto a los índices de enero y febrero que reflejaron baja calidad (ver Figura 12). 
Marco A. Aguirre, Anderson G. Rojas, Oscar B. Bermúdez, Francy V. B. Trochez

\begin{tabular}{|c|c|c|c|c|c|c|c|c|c|c|}
\hline \multicolumn{11}{|c|}{ Índices síntesis del estado fisicoquímico del agua en la Cuenca hidrográfica del río Dagua } \\
\hline Periodos & Meses & $\begin{array}{l}\text { Indice } \\
\text { Estación } \\
\text { Córdoba }\end{array}$ & $\begin{array}{l}\text { Precipitación } \\
\qquad(\mathrm{mm})^{*}\end{array}$ & $\begin{array}{l}\text { Caudal } \\
(\mathrm{m} 3 / \mathrm{s})\end{array}$ & $\begin{array}{l}\text { İndice Estación } \\
\qquad \text { Bitaco }\end{array}$ & $\begin{array}{c}\text { Precipitació } \\
\text { n (mm) })^{*}\end{array}$ & $\begin{array}{l}\text { Caudal } \\
(\mathrm{m} 3 / \mathrm{s})\end{array}$ & $\begin{array}{l}\text { Indice } \\
\text { Estación } \\
\text { Harinera }\end{array}$ & $\begin{array}{l}\text { Precipitación } \\
\qquad(\mathrm{mm})^{*}\end{array}$ & $\begin{array}{l}\text { Caudal } \\
(\mathrm{m} 3 / \mathrm{s})\end{array}$ \\
\hline \multirow{9}{*}{ 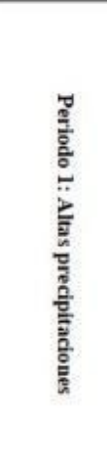 } & oct -01 & 0,01 & \multirow{3}{*}{1.224} & 60,360 & 0,33 & \multirow{3}{*}{145} & 5.238 & 0,08 & \multirow{3}{*}{145} & 3,43 \\
\hline & oct -02 & 0,06 & & 58,830 & 0,33 & & 8.715 & 0.15 & & 3,20 \\
\hline & oct -03 & 0,05 & & 65,210 & 0,37 & & 8,097 & 0,12 & & 3,11 \\
\hline & nov-01 & 0 & \multirow{3}{*}{1.032} & 60,360 & 0,26 & \multirow{3}{*}{159} & 9,872 & 0,06 & \multirow{3}{*}{159} & 4,989 \\
\hline & nov -02 & 0,09 & & 58,830 & 0 & & 8,715 & 0 & & 4,959 \\
\hline & nov -03 & 0,12 & & 65,210 & 0.25 & & 11,633 & 0 & & 5,019 \\
\hline & dic-01 & 0.3 & \multirow{3}{*}{388} & 42,40 & 0.36 & \multirow{3}{*}{136} & 9,250 & 0,42 & \multirow{3}{*}{136} & 3,060 \\
\hline & dic -02 & 0,40 & & 61,180 & 0,41 & & 10,774 & 0,35 & & 3,261 \\
\hline & dic -03 & 0.01 & & 114,15 & 0.46 & & 9,889 & 0,39 & & 3,241 \\
\hline \multirow{6}{*}{ 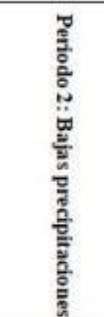 } & ene-01 & 0,37 & \multirow{3}{*}{298} & 42,98 & 0,28 & \multirow{3}{*}{134} & 9,039 & 0,38 & \multirow{3}{*}{134} & 2,761 \\
\hline & ene-02 & 0,46 & & 55,07 & 0,40 & & 9,883 & 0,42 & & 2,35 \\
\hline & ene 03 & 0,54 & & 56,40 & 0,38 & & 9,526 & 0,34 & & 2,280 \\
\hline & feb-01 & 0,87 & \multirow{3}{*}{226} & 2,286 & 0,88 & \multirow{3}{*}{47} & 6,461 & 1 & \multirow{3}{*}{47} & 23,32 \\
\hline & feb -02 & 0.91 & & 3,441 & 0.91 & & 6,485 & 0,57 & & 23,73 \\
\hline & feb -03 & 1 & & 2,460 & 1 & & 6,418 & 0,78 & & 23,04 \\
\hline
\end{tabular}

Figura 12. Índices síntesis fisicoquímico de calidad ambiental del agua ${ }^{4}$.

Fuente: Elaborado por los autores

\subsection{Actividades productivas de tipo extractivo y de servicios presentes en coberturas de la tierra}

Con base en información espacial de coberturas de la tierra suministrada por el Ideam a escala 1:100.000, se identificó que en las tres áreas de estudio definidas a través de los puntos de aforo (áreas I, II y III) el conjunto de coberturas síntesis dominante corresponde a C1 y C3 (ver Figura 13); en $\mathrm{C} 1$ se resaltan coberturas asociadas a pastos limpios, pastos enmalezados, herbazales, arbustales y mosaicos de cultivos y espacios naturales cuyas actividades productivas más importantes se vinculan a ganadería extensiva en ladera, cultivos de pastos y actividades pecuarias (avícolas y porcícolas) como se muestra la Figura 13. Respecto a las coberturas clasificadas en C3, se destacan las de bosque denso, ripario y fragmentado, así como vegetación secundaria en transición y plantaciones forestales de pino y eucalipto. Sus actividades productivas más representativas tienen diferencias marcadas entre cuenca baja y alta: en la parte alta se localiza, en menor proporción, porcicultura, avicultura y ganadería extensiva en ladera. Sin embargo, la presencia de cultivos forestales de pino y eucalipto representan una gran proporción de estas coberturas (cultivos que corresponden a la empresa Cartón de Colombia); en la cuenca baja predomina con mayor intensidad la extracción de materiales de arrastre y actividades recreativas (balnearios).

\footnotetext{
$4 *$ Precipitación mensual (CVC- 2016-2017); Oct= Octubre Nov= Noviembre Dic $=$ Diciembre Ene $=$ Enero Feb= Febrero; 1= índice de 7:00 am;2= índice de 1:00 pm;3= índice de 6:00 pm
} 
Efectos de atividades humanas extractivas y servicios recreativos sobre la calidad del agua

\begin{tabular}{|c|c|c|c|c|c|c|c|}
\hline \multirow{3}{*}{\multicolumn{2}{|c|}{$\begin{array}{l}\text { Coberturas de la tierra* } \\
\text { Area total de la cuenca }\end{array}$}} & \multicolumn{6}{|c|}{ Áreas de estudio ${ }^{* * *}$} \\
\hline & & \multicolumn{2}{|c|}{$\mathbf{I}$} & \multicolumn{2}{|c|}{ II } & \multicolumn{2}{|c|}{ III } \\
\hline & & ha & $\%$ & ha & $\%$ & ha & $\%$ \\
\hline \multirow{5}{*}{$\mathrm{C1}^{* * *}$} & Pastos limpios (ganaderia en ladera) & \multirow{5}{*}{17473,67} & \multirow{5}{*}{79,52} & \multirow{5}{*}{64512,38} & \multirow{5}{*}{76,1} & \multirow{5}{*}{74197,8} & \multirow{5}{*}{57,08} \\
\hline & Pastos enmalezados & & & & & & \\
\hline & Herbazal (herbazal denso tierra firme) & & & & & & \\
\hline & Arbustales & & & & & & \\
\hline & Mosaico de cultivos y espacios naturales & & & & & & \\
\hline $\mathrm{C}_{2}$ ** & Área construida & 79,1 & 0,36 & 292,52 & 0,35 & 302,74 & 0.23 \\
\hline \multirow{5}{*}{$\mathrm{C}^{* *}$} & Bosque denso & \multirow{5}{*}{4043,96} & \multirow{5}{*}{18,4} & \multirow{5}{*}{16149,92} & \multirow{5}{*}{19,05} & \multirow{5}{*}{51299.28} & \multirow{5}{*}{39,4} \\
\hline & Bosque fragmentado (con pastos) & & & & & & \\
\hline & Bosque ripario & & & & & & \\
\hline & Vegetación secundaria en transición & & & & & & \\
\hline & Plantación forestal (pino y eucalipto) & & & & & & \\
\hline $\mathrm{C4}^{* *}$ & $\begin{array}{l}\text { Cultivos permanentes y semipermanentes } \\
\text { (frutales citricos, café, piña y sábila) }\end{array}$ & 147,92 & 0,11 & 2738,09 & 3,23 & 2738,09 & 2,11 \\
\hline $\mathrm{C5}^{* *}$ & $\begin{array}{l}\text { Mosaico de cultivos, cultivos arbustivos } \\
\text { y transitorios y pan } \\
\text { coger (Té, caña panelera, frutales, maiz y } \\
\text { hortalizas) }\end{array}$ & 228,92 & 0,18 & 377,72 & 0,45 & 377,72 & 0,29 \\
\hline No dato & Nubes y cuerpos de agua (rios) & 0 & 0 & 704,6 & 0,83 & 1065,72 & 0,82 \\
\hline & Totales & 21973,56 & 100 & 84775,24 & 100 & 129981,35 & 100 \\
\hline
\end{tabular}

Figura 13. Coberturas de la tierra cuenca hidrográfica río Dagua 5 .

Fuente: Elaborado por los autores con datos del Ideam 2013

El conjunto de coberturas C2 corresponden en su totalidad a las áreas construidas de la cuenca, es decir centros urbanos. En ellas hay presencia de viviendas que no cuentan con sistemas de alcantarillado para sus aguas domésticas residuales, las cuales vierten directamente en fuentes hídricas. Finalmente, los conjuntos de coberturas $\mathrm{C} 4$ y $\mathrm{C} 5$ se asocian a cultivos permanentes y semipermanentes (café, frutales de cítricos, piña y sábila) y mosaico de cultivos, cultivos arbustivos, transitorios y pan coger (Té, caña panelera, frutales, maíz y hortalizas).

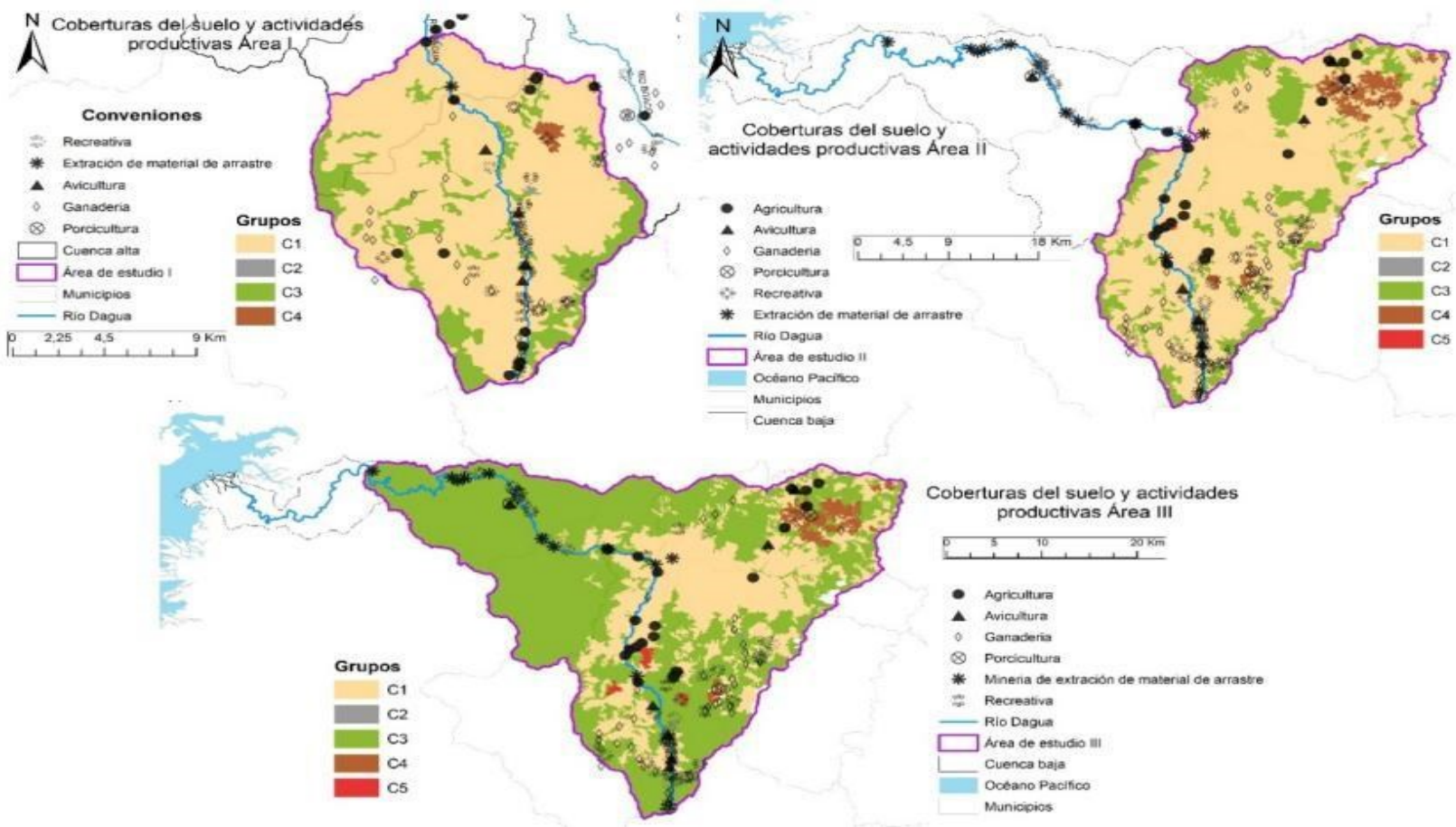

Figura 14. Coberturas de la tierra y actividades productivas extractivas y de servicios.

Fuente: Elaborado por los autores con Datos del Ideam, 2013

\footnotetext{
$5 *$ Leyenda de coberturas Corin Land Cover adaptada para Colombia a escala 1:100.000 por Ideam, 2013;
}

** Conjunto de coberturas; ***Áreas de estudio definidas para cada punto de monitoreo 


\subsection{Valoración cualitativa de la calidad ambiental del agua a través de panel de expertos}

La aplicación del panel de expertos permitió validar y complementar los resultados del índice, según la escala de valoración dispuesta en el Figura 5, evidenciando lo siguiente: Cuenca alta: actividades agrícolas y pecuarias como principales factores de afectación de la calidad ambiental del agua. Cuenca baja: minería y extracción de material de arrastre como principales factores de afectación de la calidad ambiental del agua (ver figura 15).

\begin{tabular}{|c|c|c|c|c|c|c|}
\hline & \multicolumn{2}{|c|}{ Categorías } & Agricultura & Pecuarias & Minería & Recreativas \\
\hline \multirow{2}{*}{$\begin{array}{c}\text { Cuenca } \\
\text { alta }\end{array}$} & \multirow{4}{*}{$\begin{array}{c}\text { Calidad ambiental del } \\
\text { agua }\end{array}$} & $\begin{array}{l}\text { Fauna, flora y vegetación de } \\
\text { ribera del río }\end{array}$ & & & * & \\
\hline & & Caudal, cauce y profundidad & & & & \\
\hline \multirow{2}{*}{$\begin{array}{c}\text { Cuenca } \\
\text { baja }\end{array}$} & & $\begin{array}{c}\text { Fauna, flora y vegetación de } \\
\text { ribera del río }\end{array}$ & & & & \\
\hline & & Caudal, cauce y profundidad & & & & \\
\hline \multirow{3}{*}{$\begin{array}{l}\text { Cuenca } \\
\text { alta }\end{array}$} & \multirow{7}{*}{$\begin{array}{l}\text { Factores relativos a la } \\
\text { identificación y } \\
\text { aceptabilidad de la calidad } \\
\text { del agua (cualidades } \\
\text { organolépticas) }\end{array}$} & Olor & & & * & \\
\hline & & Temperatura & & & $*$ & \\
\hline & & Transparencia & & & * & \\
\hline \multirow{4}{*}{$\begin{array}{l}\text { Cuenc } \\
\text { a baja }\end{array}$} & & & & & & \\
\hline & & Olor & & & & \\
\hline & & Temperatura & & & & \\
\hline & & Transparencia & & & & \\
\hline \multirow{2}{*}{$\begin{array}{l}\text { Cuenc } \\
\text { a alta }\end{array}$} & \multirow{4}{*}{$\begin{array}{c}\text { Factores que inciden en } \\
\text { la salud de las personas } \\
\text { y el uso del agua } \\
\text { (consumo humano) }\end{array}$} & Salud de las personas & & & & \\
\hline & & Uso del agua & & & & \\
\hline \multirow{2}{*}{$\begin{array}{l}\text { Cuenc } \\
\text { a baja }\end{array}$} & & Salud de las personas & & & & \\
\hline & & Uso del agua & & & & \\
\hline
\end{tabular}

Figura 15. Resultado valoración cualitativa. *sin presencia de la actividad productiva.

Fuente: Elaborado por los autores

\section{DISCUSIÓN DE RESULTADOS}

La discusión se centra en la correspondencia perceptiva (en términos de observación del comportamiento de las variables en las gráficas) entre los índices estimados y la presencia de actividades productivas de tipo extractivo y de servicios presentes en coberturas de la tierra. Debido a las dificultades para encontrar una correlación estadística entre los porcentajes acumulados en cada punto de monitoreo de las coberturas de la tierra y los respectivos índices síntesis fisicoquímicos, se tomó la decisión de contrastar perceptualmente los resultados a través de gráficos que relacionan los porcentajes acumulados de coberturas y los índices de calidad del agua. Los resultados del panel de expertos contribuyeron a validar las correspondencias perceptuales.

\subsection{Correspondencia perceptual entre índices síntesis fisicoquímicos del agua y coberturas $C 1$}

El conjunto de coberturas C1 (pastos limpios, enmalezados, herbazal, arbustales, mosaicos de cultivos y espacios naturales), de acuerdo con el Ideam (2013), tienen una vocación para el establecimiento y manejo de pastos para ganado bajo prácticas de manejo tecnológico asociadas a limpieza o fertilización, entre otras. En la cuenca del río Dagua, este conjunto de coberturas se relacionó con el desarrollo de actividades extractivas como la ganadería, avicultura y porcicultura que, de acuerdo con la valoración perceptiva del panel de expertos, implican grados de afectación 
negativos en la calidad ambiental del agua, sus condiciones organolépticas, uso y salud de las personas.

Del mismo modo, de acuerdo con la Figura 16, aguas arriba de los puntos de monitoreo La Harinera y Bitaco en la cuenca alta del río Dagua, donde predomina el conjunto de coberturas de tipo $\mathrm{C} 1$, el índice refleja un estado físicoquímico de calidad ambiental medio y bajo. Los índices, a su vez, se consolidan en estos rangos en la medida que disminuyen las precipitaciones y caudales en la cuenca (ver Figura 13).

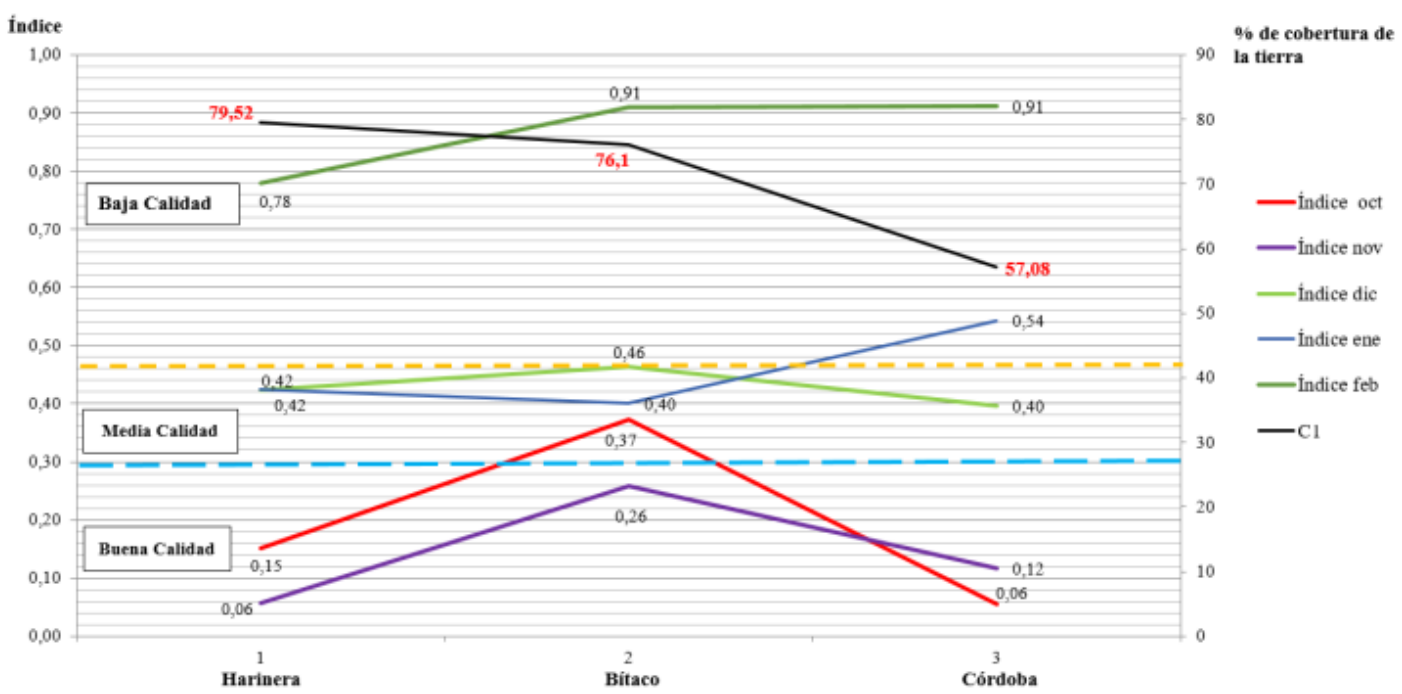

Figura 16. Relación entre el índice y conjunto de coberturas $\mathrm{C} 1$

Fuente: Elaborado por los autores

Lo anterior, según las actividades antrópicas identificadas en campo asociadas a ganadería en ladera, deforestación, captación directa de agua para procesos pastoriles, así como el constante vertimiento de aguas residuales domésticas y agrícolas y residuos (sólidos y orgánicos) de manera directa a cuerpos de agua, coincide con los resultados encontrados en el panel de expertos (ver Figura 17).

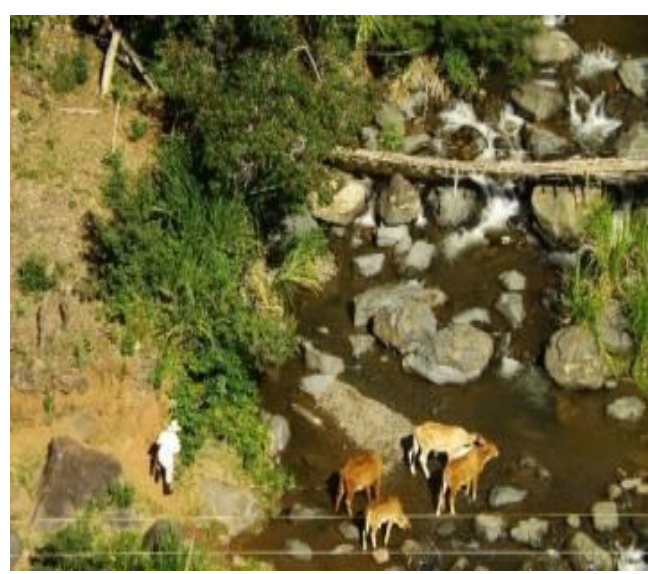

Figura 17. Presencia de ganadería, cauce principal del río Dagua (Cuenca alta).

Fuente: Los autores

Por su parte, en la cuenca baja del río Dagua, sobre el punto de monitoreo Córdoba, el porcentaje de coberturas $\mathrm{C} 1$ es menor en comparación a la cuenca alta. Ello se evidencia en baja presencia agropecuaria. Dicho factor espacial se relaciona con los resultados del panel de expertos, el cual indica que la afectación de la calidad ambiental del agua no se asocia este tipo de actividades. No obstante, los agentes sociales señalan que, aunque existen unidades productivas familiares dedicados a la agricultura, estas son principalmente de autosustento, compuestas por 
cultivos de pancoger y frutales, los cuales no implican la realización de procesos intensivos o tecnificados tal como: control de plagas, fertilización química, riego, entre otros. Estos elementos coinciden con la no correspondencia perceptual entre el conjunto de coberturas $\mathrm{C} 1$ y el estado fisicoquímico del agua reflejado en la Figura 16.

\subsection{Correspondencia perceptual entre índices síntesis fisicoquímicos del agua y coberturas C2 у C3}

El conjunto de coberturas $\mathrm{C} 2$ corresponde a áreas construidas y, C3, a bosques naturales y plantados, las cuales, si bien no se asocian directamente con una actividad productiva, sí presentan correspondencia con el comportamiento de los índices de calidad del agua. Las coberturas C2 tienen mayor proporción espacial en la cuenca alta. Su correspondencia perceptual con bajos niveles de calidad ambiental del agua es alta, específicamente en los puntos de monitoreo Bitaco y Dagua, durante los meses de diciembre, enero y febrero tal como se muestra en la Figura 18. Dicha variabilidad, según los datos recabados en campo y a través del panel de expertos, se asocia con la presencia de residuos y vertimientos domésticos provenientes de centros poblados, aumento de la población y la construcción de viviendas de recreo.

Por su parte, en la cuenca baja, para los meses de octubre y noviembre, donde las precipitaciones son altas, se presenta una buena calidad ambiental del agua; contrario a esto, entre los meses de diciembre a febrero (meses de menor precipitación) la calidad pasó de media a baja. En la Figura 19 se evidencia parte de las actividades humanas que afectan la calidad ambiental del agua.

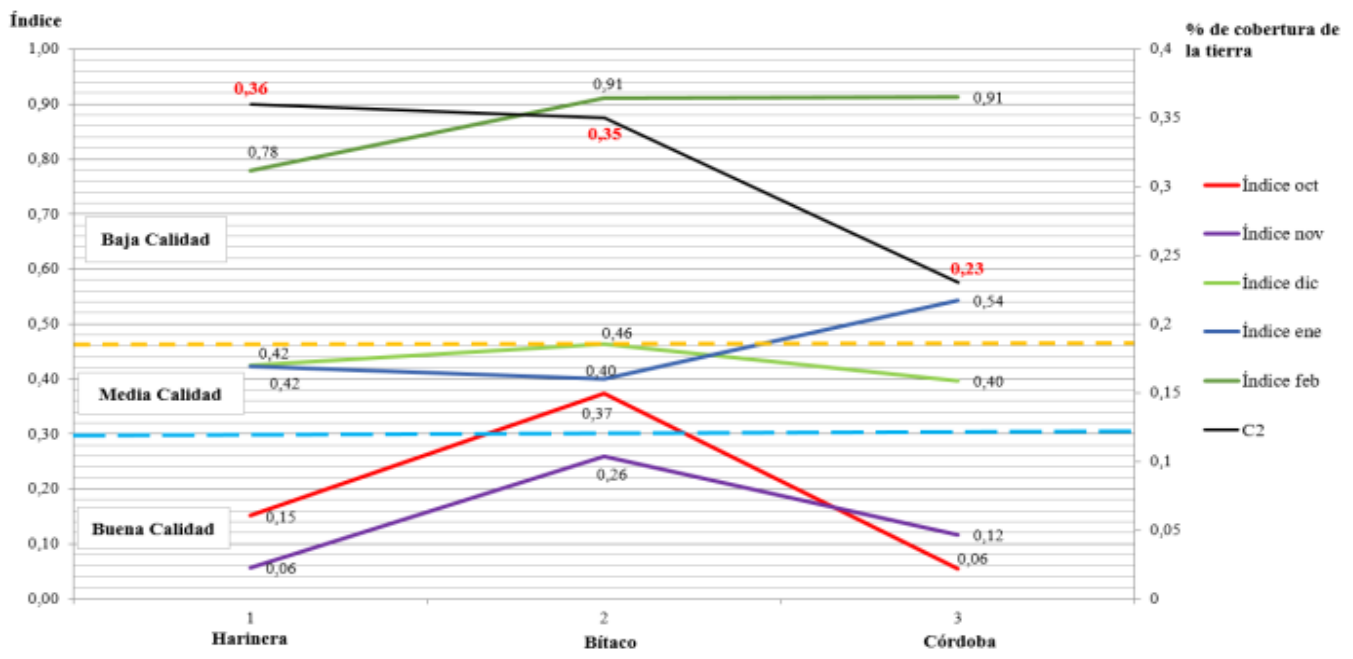

Figura 18. Relación entre el índice síntesis fisicoquímico y el conjunto de coberturas C2. Fuente: Elaborado por los autores

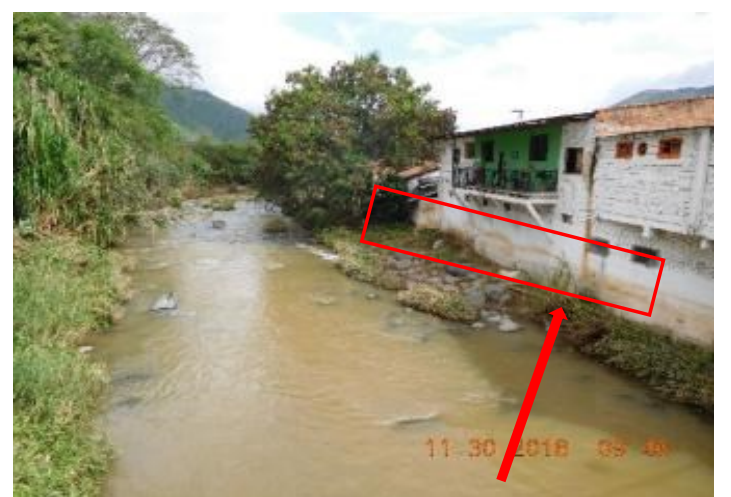

Figura 19. Descarga de aguas domésticas en el cauce principal del río Dagua, parte alta de la cuenca Fuente: Autores 
Respecto al conjunto de coberturas de la tierra $\mathrm{C} 3$, asociadas principalmente con áreas boscosas y plantaciones forestales de pino y eucalipto (Ideam, 2013), la Figura 20 muestra que en los tres puntos de monitoreo no existe correspondencia perceptual respecto a calidad ambiental e incremento de coberturas. Pese a una mayor acumulación de coberturas C3 en el área de estudio III (estación Córdoba), los índices de calidad ambiental del agua reflejan tendencias a su mala calidad. Ello tiene un grado de relación con las bajas precipitaciones registradas en la cuenca y la disminución de caudales (ver Figura 12).

Los resultados del panel de expertos y el trabajo en campo permitieron contrastar los datos obtenidos en cada uno de los índices a partir de cuatro aspectos clave: primero, a pesar de existir espacialmente una significativa proporción de cobertura C3 en la cuenca, en ellas se identifica el desarrollo de actividades productivas de tipo extractivo como son la ganadería en ladera, monocultivos de piña y sábila, los cuales conllevan a la ampliación de la frontera agrícola; segundo, la ausencia de sistemas de alcantarillado, plantas de tratamiento de aguas domésticas y pozos sépticos en la cuenca, incide en la calidad ambiental del agua y en los procesos de degradación ambiental del conjunto de coberturas C3; tercero, la cuenca es objeto de procesos intensivos de deforestación en importantes áreas de reserva (Ley Segunda de 1959); y cuarto, extensas áreas en cultivos forestales de pino y eucalipto localizadas principalmente en la parte alta de la cuenca, las cuales pertenecen a la empresa Cartón Colombia, quien ha hecho uso de dicha actividad extractiva por más de 50 años en la cuenca (Aguirre et al. 2017; Campo y Rojas, 2018; Universidad del Valle, 2016a; 2016b).

Finalmente, con respecto al conjunto de coberturas C3 en la parte baja de la cuenca, se desarrollan actividades productivas extractivas (extracción de material de arrastre y minería aurífera ilegal y artesanal) y de servicios (balnearios y puntos estratégicos localizados sobre la vía principal de la cuenca para el lavado de tracto camiones) de manera no planificada. Respecto a la minería para extracción de materiales de arrastre, en la cuenca baja, se resalta la degradación ambiental acelerada del paisaje y las coberturas C3, producto de la ampliación de la doble calzada Lobo Guerrero-Buenaventura, vía que comunica al centro del país con el principal puerto marítimo de Colombia, localizado en la parte baja de la cuenca. A continuación, en las Figuras 21 y 22 se evidencia parte de los elementos encontrados en campo asociados a la calidad ambiental del agua.

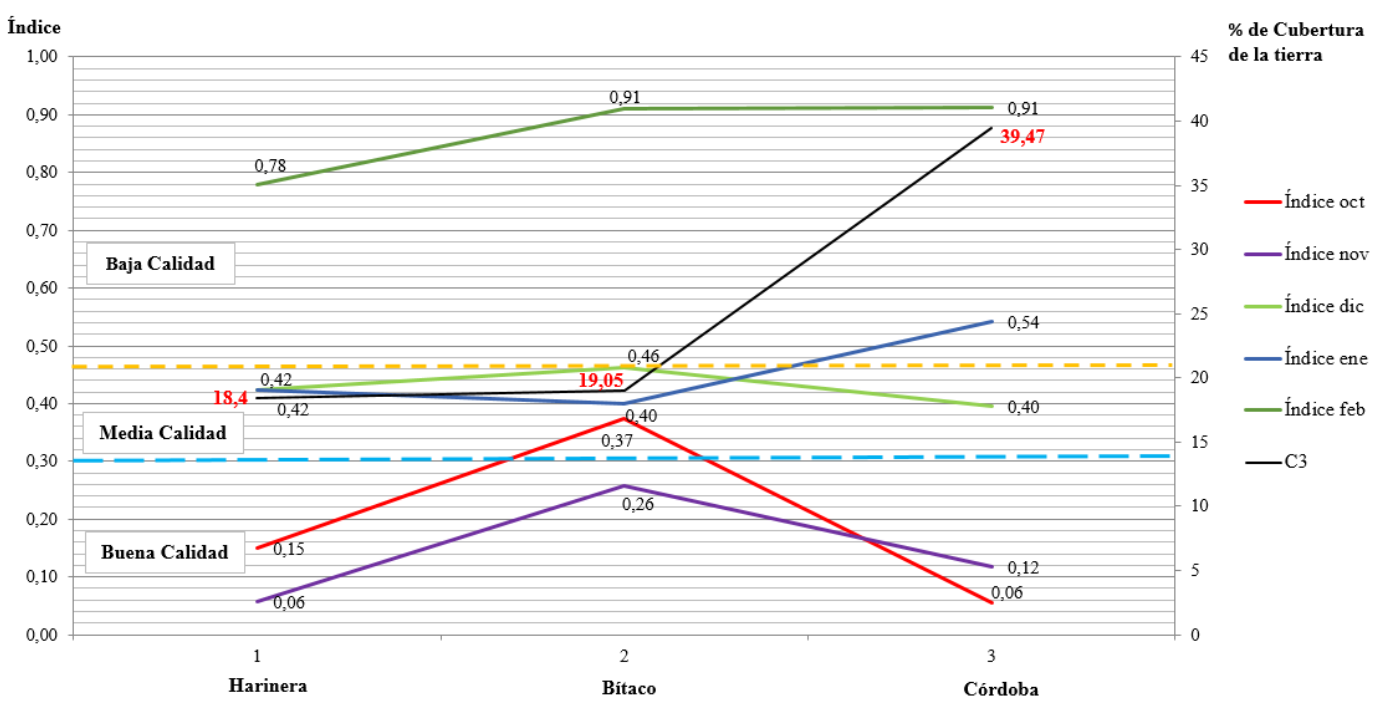

Figura 20. Relación entre el índice síntesis fisicoquímico y el conjunto de coberturas C3.

Fuente: Elaborado por los autores 
Marco A. Aguirre, Anderson G. Rojas, Oscar B. Bermúdez, Francy V. B. Trochez

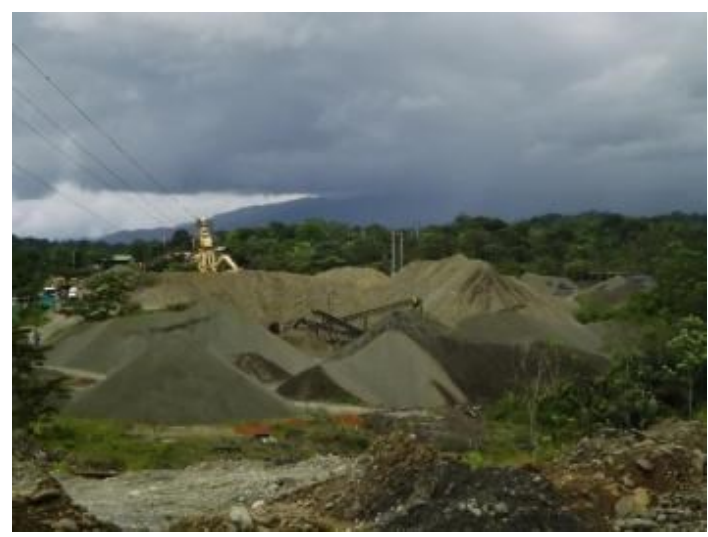

Figura 21. Extracción de material de arrastre en Loboguerrero, parte alta de la cuenca.

Fuente: Autores

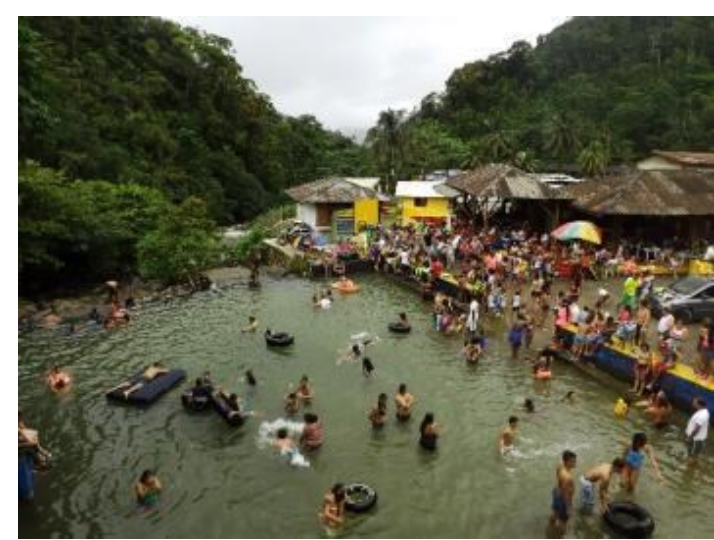

Figura 22. Actividades recreativas asociadas a balnearios, parte baja de la cuenca.

Fuente: Autores

\subsection{Correspondencia perceptual entre índices síntesis fisicoquímicos del agua y coberturas C4 у C5}

En este apartado se expondrán las correspondencias perceptuales entre el estado físicoquímico del agua y los conjuntos de coberturas de la tierra $\mathrm{C} 4$ y $\mathrm{C} 5$. El primer conjunto se relaciona con coberturas de cultivos permanentes y semipermanentes. En ellos se identifica plantaciones de frutales (en especial cítricos), café, piña y sábila en áreas de altas pendientes. Dichas coberturas predominan espacialmente en la parte alta de la cuenca. El segundo conjunto de coberturas comprende mosaicos de cultivos (pastos, cultivos permanentes y semi-permanentes) y cultivos transitorios como son caña panelera, piña, cultivos de té (a gran escala) y frutales (cítricos), así como cultivos pancoger, asociados a maíz, hortalizas y frutales. A diferencia de las coberturas $\mathrm{C} 4$, éstas se distribuyen por toda la cuenca. Para ambos conjuntos de coberturas, mediante trabajo en campo y con información secundaria, se identificó que en la cuenca se emplean insumos químicos sin controles respectivos en su desarrollo.

Según la Figura 17 se evidencia un grado de correspondencia perceptual entre los índices (valores de media y baja calidad ambiental del agua) y el incremento de coberturas de la tierra en el punto de monitoreo Bitaco. Dicho comportamiento se presentó en los meses de menor precipitación y disminución de caudales en la cuenca (ver Figura 12). Respecto al punto de monitoreo localizado en Córdoba, se esperaría que al disminuir el porcentaje de este conjunto de coberturas (teniendo en cuenta una disminución de las actividades antrópicas) los índices no reflejaran comportamientos como los presentes en la Figura 23. De acuerdo al panel de expertos, las actividades agrícolas en la cuenca afectan negativamente la calidad ambiental del agua en aspectos como la salud de las personas, las características organolépticas y otros usos que dependan de ella (ver Figura 15). En la Figura 24 se perciben algunos elementos asociados a prácticas productivas en la cuenca. 


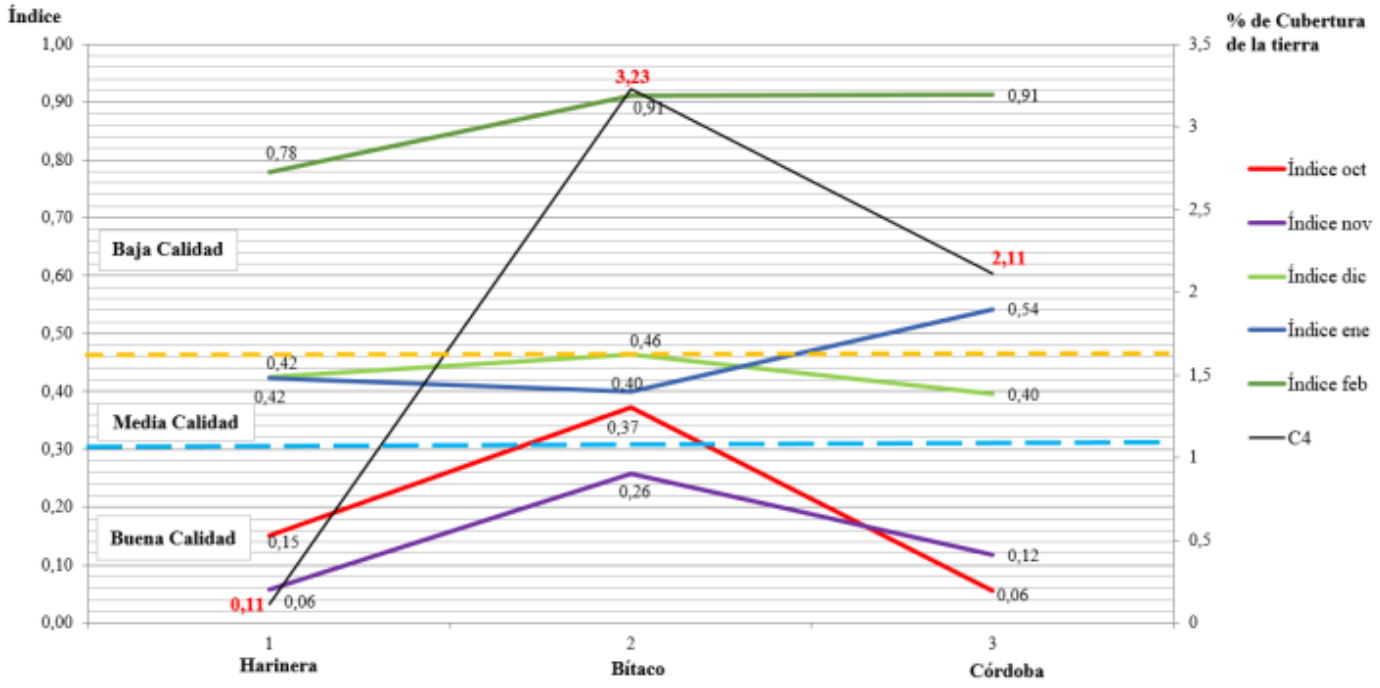

Figura 23. Relación entre el índice síntesis fisicoquímico y el conjunto de coberturas C4.

Fuente: Elaborado por los autores

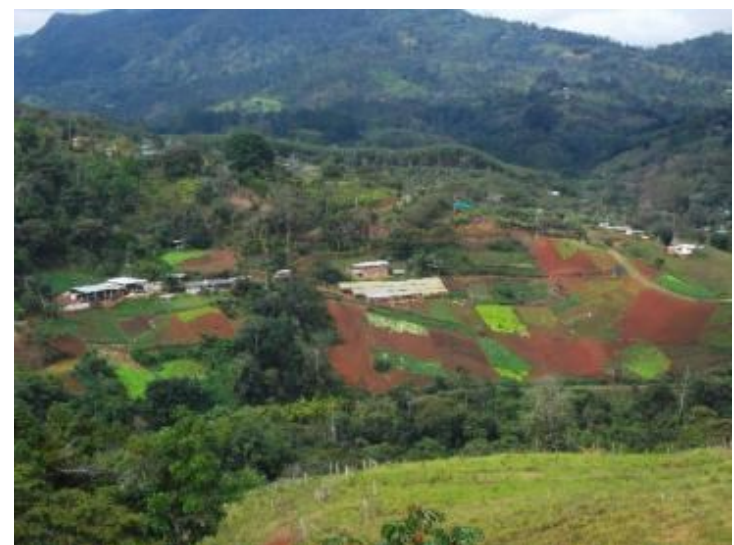

Figura 24. Mosaico de cultivos (hortalizas, café, plátano y caña panelera), parte alta de la cuenca (uso intensivo de agroquímicos).

Fuente: Autores

Con respecto al conjunto de coberturas C5, se evidenció correspondencia perceptual directa entre porcentajes de cobertura e índices de calidad ambiental del agua a partir de los resultados obtenidos para la estación de monitoreo Bitaco (ver Figura 25). Los índices presentaron tendencias asociadas a mediana y mala calidad del agua. Por otro lado, tal como ocurre en las gráficas anteriores, los índices se relacionan con bajas precipitaciones y disminución de caudales. Con respecto a las estaciones de La Harinera y Córdoba, se esperaría que al disminuir el porcentaje de coberturas $\mathrm{C} 5$, los índices reflejarán mejor calidad del agua. No obstante, los resultados indican baja y mala calidad.

A pesar de haber tenido dificultades para desarrollar una metodología soportada estadísticamente, con base en los resultados del panel de expertos y trabajo en campo, es posible afirmar que existe afectación de las actividades humanas de tipo extractivo (agricultura y ganadería en ladera y minería extractiva de material de arrastre) presentes en las coberturas C5, en la calidad ambiental del agua. 


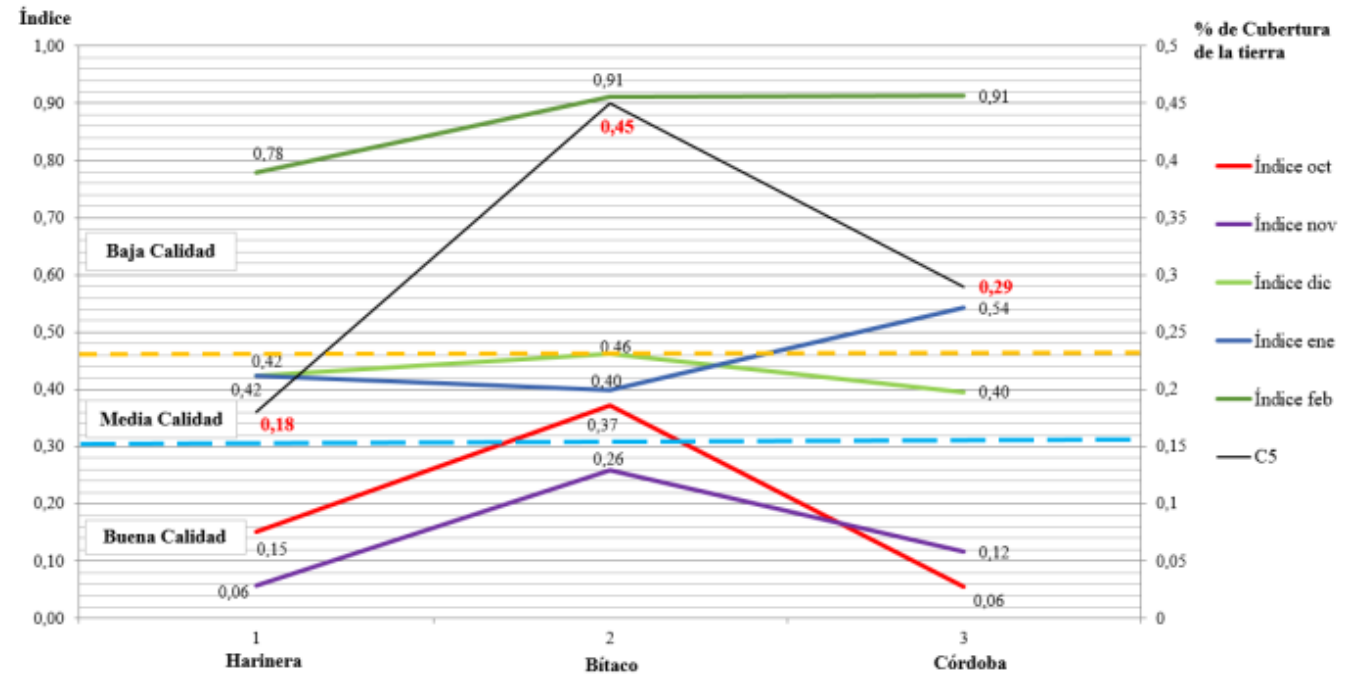

Figuras 25. Relación entre el índice síntesis fisicoquímico y el conjunto de coberturas C5. Fuente: Elaborado por los autores

\section{CONCLUSIONES}

Con los resultados obtenidos tanto de la correspondencia perceptiva entre actividades productivas presentes en coberturas de la tierra con los índices de calidad ambiental del agua, y la información suministrada por agentes sociales a través de paneles de expertos se identificó que, en la cuenca alta, las actividades de tipo extractivo como son ganadería extensiva en ladera, agricultura tecnificada, así como actividades de servicios asociadas a la oferta de viviendas campestres para recreación y esparcimiento, afectan la calidad ambiental y disponibilidad del agua en la cuenca hidrográfica. Sumado a esto, durante el trabajo de campo y los aportes de los agentes sociales participantes de esta investigación se identificó focos de contaminación difusa asociados con ausencia de sistemas de alcantarillado y saneamiento básico (recolección de residuos sólidos y orgánicos) en los centros poblados ubicados en la cuenca hidrográfica. Situación que aumenta la afectación de la calidad del agua.

En la cuenca baja, la correspondencia perceptual evidenció que la minería extractiva (material de arrastre) es la principal actividad que afecta la calidad ambiental del agua. Sin embargo, los agentes sociales a través del panel de expertos reconocen otro factor que aumenta la contaminación del vital líquido asociado al desarrollo de actividades agropecuarias presentes en la cuenca alta. Del mismo modo, los agentes señalan que las actividades de servicios recreativos en la cuenca hidrográfica baja no representan un problema significativo en la calidad ambiental del agua, aunque suscita preocupaciones debido a que su aumento paulatino demanda mayor uso del agua, y con el tiempo, podría conllevar a problemas de contaminación por el desarrollo de actividades turísticas. Adicionalmente, los agentes sociales destacan desde su experiencia que los bajos niveles de precipitación del ciclo natural del agua que determinan los caudales, representan un factor clave para evidenciar condiciones de baja calidad ambiental del agua en la cuenca hidrográfica.

Los resultados del trabajo contribuyen con su metodología de tipo mixto de la siguiente forma: primero, con argumentos científicos que sirven de base e insumo en procesos de gestión del agua de las comunidades locales; y segundo, se da un paso importante con respecto a los estudios ambientales en cuencas hidrográficas en contextos como el de Colombia, donde existen limitantes asociadas al acceso de información primaria y recursos económicos por parte del Estado.

Finalmente, para mejorar la precisión de estudios que relacionan la calidad ambiental del agua con el desarrollo de actividades humanas y con base en los antecedentes de la investigación, es necesario contar con sistemas de monitoreo ambiental que constantemente registren parámetros fisicoquímicos, biológicos y actividades antrópicas presentes en coberturas de la tierra entre otros elemento asociados con la inclusión y participación de las comunidades. 


\section{REFERENTES}

Acosta, Y. (2015). Impacto de la cobertura del suelo sobre la calidad del agua en el cauce medio y bajo del río Pance. Colombia. Universidad del Valle. Departamento de Geografía. $124 \mathrm{p}$

Ahearn, D. S., Sheibley, R. W., Dahlgren, R. A., Anderson, M., Johnson, J., \& Tate, K. W. (2005).

Land use and land cover influence on water quality in the last free-flowing river draining the western Sierra Nevada, California. Journal of Hydrology, 313(3-4) 234-247

Aguirre, A., López, L., Bolaños, F., González, D., y Buitrago O. (2017). Percepción del paisaje, agua y ecosistemas en la cuenca del río Dagua, Valle del Cauca, Colombia. Perspectiva Geográfica, 22(1)

Andrade G. I., M. E. Chaves, G., y Corzo, C. (2018). Transiciones socioecológicas hacia la sostenibilidad. Gestión de la biodiversidad en los procesos de cambio en el territorio continental colombiano. Primera aproximación. Bogotá: Instituto de Investigación de Recursos Biológicos Alexander von Humboldt. 220 p.

Arruebo, T., Pardo, A., Rodríguez F., Lajana, J., y Del Valle, J. (2009). Método específico para la evaluación medioambiental de los lagos de origen glaciar pirenaicos y su aplicación al lago de Sabocos. Pirineos. 164, (140). Recovered in: 20.jun.2020, de $<$ http://pirineos.revistas.csic.es/index.php/pirineos/article/view/33/34>

Auquilla Cisneros, R. (2005). Uso del suelo y calidad del agua en quebradas de fincas con sistemas silvopastoriles en la subcuenca del Río Jabonal. Catie, Turrialba, Costa Rica.

Benez, M, C. (2009). Percepciones ambientales de la calidad del agua superficial en la microcuenca del río Fogótico, Chiapas: Sobre el concepto de percepción: El complejo proceso de la relación ser humano-ambiente. Frontera Norte. 22, (43)

Billen, G., Garnier, J., Ficht, A., \& Cun, C. (2011). Modeling the response of water quality in the seine river estuary to human activity in its watershed over the last 50 years. Estuaries. 4, (6B).

Recovered in 20.jun.2020, de

https://www.researchgate.net/profile/Josette_Garnier/publication/226317483_Modeling_the_Respo nse_of_Water_Quality_in_the_Seine_River_Estuary_to_Human_Activity_in_Its_Watershed_over the_Last_50_Years/links/5549flba0cf205bce7ac5305.pdf

Boelee, E \& Laamrani, H. (2004). Multiple use of irrigation water in Northeastern Morocco. En:

Beyond Domestic: Case studies on poverty and productive uses of water at the household level. $\mathrm{N}^{\circ}$ 41. Recovered in:20 jun.2020, de

http://www.protos.be/sites/default/files/library_assets/W_PRO_20_E8_Beyond_Domestic.pdf\#page $=120$

Bu, H., Meng, W., \& Zhang, Y. (2014). Spatial and seasonal characteristics of river water chemistry in the Taizi River in Northeast China. Environmental monitoring and assessment, 186(6), 36193632.

Camdessus, M., Badré, B., Chéret, I., Frédéric, P., Buchot, T., \& Hulsz, L. (2006). Água para todos. Traducción por el Fondo de Cultura de México. 294pp.

Carstens, D \& Amer, R. (2018). Spatio-temporal analysis of urban changes and surface water quality. Journal of hydrology, 569 (2019) 720-734.

Castro, E. (2007). Water governance in the twentieth-first century. Revista Ambiente \& Sociedade, Campinas, 10(2), 97-118

Cirelli, P. \& Du Mortier, C. (2005). Evaluación de la condición del agua para consumo humano en Latinoamérica. Tecnologías solares para la desinfección y descontaminación del agua. Solar Safe Water, 11-26. 
Collingwood, G. (2006). Idea de la naturaleza. (2 ${ }^{\mathrm{a}}$ Edición). Fondo de Cultura Económica, México D. F. 243 pp.

Corporación Autónoma Regional del Valle del Cauca -CVC-. (2011). Protocolo de información ambiental. CVC, Dirección Técnica Ambiental, Valle del Cauca, Colombia. 26pp. Recovered in:20 jun.2020, de: http://www.geo.cvc.gov.co/pdf/CoberturaUso.pdf $\overline{\text { del Cauca. }}$

(s. f.). Fichas ilustrativas de la situación ambiental de las cuencas hidrográficas del Valle

Houngbo, F. (2018). El papel de ONU: agua como mecanismo de coordinación interinstitucional para el agua y el saneamiento. Organización de las Naciones Unidas, 55(1). Recovered in 20 junh.2020, de: https://unchronicle.un.org/es/article/el-papel-de-onu-agua-como-mecanismo-decoordinaci-n-interinstitucional-para-el-agua-y-el

Escofier, B, y Pagés, J. (1992). Análisis factoriales simples y múltiples. objetivos, métodos e interpretación. Servicio editorial de la Universidad de País Vasco.

Escobar, L. (2008). Indicadores ambientales sintéticos: una aproximación conceptual desde la estadística multivariante [En línea]. Gestión y ambiente, 11(1), 130.

Fernandez, N y Solano, F. (2009). Índices de calidad y de contaminación del Agua como herramienta para la gestión de los recursos hídricos. Medio Ambiente y Desarrollo.(16)

Ferrer, D., \& Muñoz, I. (2013). Hidromorfología fluvial: algunos apuntes aplicados a la restauración de ríos en la cuenca del Duero. Ministerio de Agricultura, Alimentación y Medio Ambiente. Madrid: Confederación Hidrográfica del Duero.

Gyawali, S., Techato, K., Monprapussorn, S., \& Yuangyai, C. (2013). Integrating land use and water quality for environmental based land use planning for U-tapao river basin, Thailand.

Procedia-Social and Behavioral Sciences, 91, 556-563.

Instituto de Hidrología, Meteorología y Estudios Ambientales -Ideam-. (1998). El medio ambiente en Colombia. Ministerio del Medio Ambiente, Bogotá. p25.

(2013). Catálogo de Patrones de Coberturas de la Tierra Colombia. [Consultado en Junio 2017]. Recovered in 20 jun.2020, de en http://www.Ideam.gov.co/documents/11769/153716/Catalogo+Coberturas+Tierra.pdf/f2eafe32f300-4ae7-9ab7-f90a8670d75e

Li, S., Gu, S., Liu, W., Han, H., \& Zhang, Q. (2008). Water quality in relation to land use and land cover in the upper Han River Basin, China. Catena, 75(2), 216-222.

Leff Z. E. (2007). La complejidad ambiental: del logos científico al dialogo de saberes. En: Las ciencias ambientales como nueva área del conocimiento para Colombia. Rcfa - Colciencias, Bogotá, Colombia. 53-65.

Loaiza, Y., y Osorio A. (2009). Gestión del agua en el sector de la ganadería bovina en la cuenca Río la Vieja departamentos de Quindio y Risaralda. Universidad Tecnológica de Pereira. 97 pp.

Lobato, R. (2014). Sobre agentes sociais, escala e produção do espaço: um texto para discussão. $A$ produção do espaço urbano: agentes e processos, escalas e desafios, 41- 51. São Paulo, Brasil: Contexto.

Londoño, C. (2001). Cuencas hidrográficas: bases conceptuales-caracterización planificaciónadministración. Ibagué: Universidad del Tolima, Facultad de Ingeniería Forestal, 2001. 359 pp. Recuperado de

$<$ http://www.ut.edu.co/academi/images/archivos/Fac_Forestal/Documentos/LIBROS/cuencas\%20hi 
Efectos de atividades humanas extractivas y servicios recreativos sobre la calidad del agua

drograficas $\% 20$ bases $\% 20$ conceptuales $\% 20 \% 20$ caracterizacion $\% 20 \% 20$ planificacion $\% 20$ yorganiza cion\%20-\%20CARLOS\%20LONDOO.pdf>

Lussault, M. (2015). El hombre espacial: la construcción social del espacio humano. Amorrotu Editores, Buenos Aires-Madrid, 345.

Martínez, C. (2012). El muestreo en investigación cualitativa: principios básicos y algunas controversias. Revista Ciencia \& Saúde Colectiva, Rio de Janeiro. 17(3), 613-619.

Mc Junkin, E. (1988). Agua y salud humana. Noriega Editores, México D.F Traducción al español de Edward Cruz Quevedo, Lima, Perú.

Medeiros, A.C., Freitas Faial, K.R., Freitas Faial, K.D., Da Silva Lopes, I.D., De Oliveira Lima, M., Guimarães, R. M., y Mendonça, N. M. (2017). Quality index of the surface water of Amazonian rivers in industrial areas in Pará, Brazil. Marine Pollution Bulletin, 123(1-2), 156-164.

Naciones Unidas (2010). Resolución aprobada por la Asamblea General el 28 de julio de 2010. (A/64/L.63/Rev.1 y Add.1). 64/292. El derecho humano al agua y el saneamiento, 3.

Organización Mundial de la Salud. (2006). Guías para la calidad del agua potable: OMS. 3 ed. Vol. 1. Suiza. [consultado marzo 2019]. Recovered in 20 jun.2020, de < http://www.who.int/water_sanitation_health/dwq/gdwq3_es_fulll_lowsres.pdf?ua=1>

Pérez, J., Nardini, A., y Galindo, A. (2018). Análisis comparativo de índices de calidad del agua aplicados al Río Ranchería, La Guajira-Colombia. Información Tecnológica,29(3), 47-58.

Rojas A y Campo, L. (2018). Valoración de la calidad del agua a partir de la visión de agentes sociales en la cuenca hidrográfica del río Dagua. Entorno GeográFico, (16).

Robledo, J., Vanegas Chacón, E. A., \& García Álvarez, N. (2014). Calidad del agua del río Túnico como respuesta al uso del suelo. Revista Ciencias Técnicas Agropecuarias, 23 (3), 41-45.

Samboni, N., Carvajal, Y., \& Escobar, J. (2007). Revisión de parámetros fisicoquímicos como indicadores de calidad y contaminación del agua. Revista Ingeniería e Investigación 27 (3): 172-181

Segura, T.L. (2007). Estudio de antecedentes sobre la contaminación hídrica en Colombia. Escuela superior de Administración Pública ESAP, Bogotá D.C,133.

Senzanje, A., Rusere, S., \& Boelee, E. (2008). Multiple use of water and water productivity of communal small dams in the Limpopo Basin, Zimbabwe. Irrigation and Drainage Systems. 22, (3).

Sutadian, A.D., Muttil, N., Yilmaz, A.G., \& Perera, B.J.C. (2016). Development of river water quality indices-a review. Environ. Monit (188), 58.

Tomaselli, M.O. (2013). Actividades Antropogénicas y su incidencia en la calidad del agua del Río Santa Rosa, sector el Playón- La Avanzada. Guayaquil, Ecuador. Universidad de Guayaquil.

Tyagi, S., Sharma, B., Singh, P., \& Dobhal, R. (2013). Water Quality Assessment in Terms of Water Quality Index. American Journal of Water Resources, 1(3), 34-38.

Universidad del Valle (2016a). Informe avance final marco teórico y metodológico actividad 1.

Proyecto de Investigación Estrategias para la Recuperación y Manejo Integrado del Recurso Hídrico en las Cuencas del Cauca y Dagua, en el Valle del Cauca. En proceso editorial.

(2016b). Informe diagnóstico de la gestión del agua en la cuenca del río Dagua.

Proyecto de Investigación Estrategias para la Recuperación y Manejo Integrado del Recurso Hídrico en las Cuencas del Cauca y Dagua, en el Valle del Cauca. En proceso editorial.

Zhao, J., Lin, L., Yang, K., Liu, Q., \& Qian, G. (2015). Influences of land use on water quality in a reticular river network area: a case study in Shanghai, China. Landscape and Urban Planning, 137, 20-29. 
Marco A. Aguirre, Anderson G. Rojas, Oscar B. Bermúdez, Francy V. B. Trochez

Zhou, P., Huang, J., Pontius Jr, R. G., \& Hong, H. (2016). New insight into the correlations between land use and water quality in a coastal watershed of China: does point source pollution weaken it? Science of The Total Environment, 543, 591-600.

\section{Leyes y decretos}

Decreto n. 1729 de 2002 - Cuencas hidrográficas Recuperado em 20.jun.2020, de:

http://www.Ideam.gov.co/documents/24024/36843/Decreto_1729_de_2002.pdf/59ad8528-11794fd7-9075-aed67fce 2 b40

Ley $2^{d a}$ de1959. Sobre economía forestal de la Nación y conservación de recursos naturales renovables. Recovered in 20.jun.2020, de en:

http://www.Ideam.gov.co/documents/24024/26915/C_Users_hbarahona_Desktop_Monica+R_norm as+pag+web_ley+2+de+1959.pdf/11 ec7647-b090-4ce-2-b863-00b27766ёdf8 